Comment. Math. Helv. 73 (1998) 637-676

(C) 1998 Birkhäuser Verlag, Basel

$0010-2571 / 98 / 040637-40 \$ 1.50+0.20 / 0$

Commentarii Mathematici Helvetici

\title{
Cogroups in algebras over an operad are free algebras
}

\author{
Benoit Fresse
}

\begin{abstract}
Let $\mathcal{P}$ be an operad defined over a field of characteristic zero. Let $R$ be a cogroup in the category of complete $\mathcal{P}$-algebras. In this article, we show that $R$ is necessarily the completion of a free $\mathcal{P}$-algebra. We also handle the case of cogroups in connected graded algebras over an operad, and the case of groups in connected graded coalgebras over an operad.
\end{abstract}

Mathematics Subject Classification (1991). 16W30, 18C15, 17A30, $18 \mathrm{D} 10$.

Keywords. Cogroup, operad.

\section{Introduction}

Fix a ground field of characteristic 0 . If $H$ is a complete commutative Hopf algebra, then it is known that $H$ is necessarily a symmetric algebra (and thus, $H$ is a free commutative algebra). Dually, if $H$ is a connected cocommutative Hopf algebra, then by the Milnor-Moore theorem, $H$ is the enveloping algebra of some Lie algebra $L$, and by the Poincaré-Birkhoff-Witt theorem, as a coalgebra, $H$ is isomorphic to the symmetric algebra $S(L)$. Finally, $H$ is a cofree cocommutative coalgebra.

The purpose of this article is to extend these assertions to other kinds of algebras (or coalgebras). More precisely, we consider algebras over a fixed unital operad $\mathcal{P}$ (see 1.1.5, 1.1.6), such as commutative algebras for $\mathcal{P}=\mathcal{C}$ om, Lie algebras for $\mathcal{P}=\mathcal{L} i e$, associative algebras for $\mathcal{P}=\mathcal{A} s$, etc. The category of complete $\mathcal{P}$-algebras (cf. 1.1.11) is equipped with a coproduct denoted by $\vee$. A cogroup object in this category is a complete $\mathcal{P}$-algebra $R$ together with a "coproduct" $\gamma: R \longrightarrow R \vee R$, and an "antipode" $\iota: R \longrightarrow R$, which satisfy the usual identities (cf. definition 1.2.1). As an example, in the case of commutative algebras, $\vee$ is given by the tensor product, and a cogroup object is a complete commutative Hopf algebra. We prove the following theorem.

0.1. Theorem. Fix a ground field of characteristic 0 . Let $\mathcal{P}$ be a unital operad. If $R$ is a complete $\mathcal{P}$-algebra equipped with a cogroup structure, then $R$ is the 
completion of a free $\mathcal{P}$-algebra.

This theorem has a graded version. Since a connected graded $\mathcal{P}$-algebra turns out to be a complete graded $\mathcal{P}$-algebra, we obtain in particular:

0.2. Theorem. Fix a ground field of characteristic 0 . Let $\mathcal{P}$ be a unital operad. If $R$ is a connected graded $\mathcal{P}$-algebra equipped with a cogroup structure, then $R$ is a free graded $\mathcal{P}$-algebra.

With an additional finiteness assumption, it is possible to prove the dual statement:

0.3. Theorem. Fix a ground field of characteristic 0 . Let $\mathcal{P}$ be a unital operad. Assume that each $k$-module $\mathcal{P}(n)$ is finite dimensional. If $C$ is a connected graded $\mathcal{P}$-coalgebra equipped with a group structure, then $C$ is a cofree graded $\mathcal{P}$-coalgebra.

Here are some classical instances of theorems 0.1 and 0.2 .

Let $\mathcal{P}=\mathcal{C}$ om be the operad of commutative algebras (without unit). As mentioned above, a cogroup in the category of complete commutative algebras is nothing but a complete commutative Hopf algebra, and is also known as a formal group. Dually, a group in the category of connected cocommutative coalgebras is nothing but a connected cocommutative Hopf algebra. In this case, theorem 0.1 is due to Cartier (cf. [2]), and, as explained in the beginning of this introduction, theorem 0.3 follows from the Milnor-Moore theorem and the Poincaré-BirkhoffWitt theorem.

The case $\mathcal{P}=\mathcal{A} s$, the operad of associative algebras (without unit) is handled by I. Berstein in [1].

In the case $\mathcal{P}=\mathcal{L} i e$, the theorems above do not seem to be stated explicitly in the litterature. Theorems 0.1 and 0.2 give the following statement

0.4. Theorem. Assume the ground field $k$ to be of characteristic zero. If $L$ is a pronilpotent Lie algebra (resp. a connected graded Lie algebra) equipped with a cogroup structure, then $L$ is the completion of a free Lie algebra (resp. a free graded Lie algebra).

The case $\mathcal{P}=\mathcal{L} e i b^{!}$, the operad of Leibniz dual algebras is handled by J.M. Oudom in [19]. In fact, theorem 0.2 answers a question asked in [19].

In the first section of this paper, we provide a brief summary of the algebraic operad theory, and we introduce the notion of a cogroup. 
In the second section, we introduce a symmetric monoidal category, whose objects are called right $\mathcal{P}$-modules. We call cocommutative $\mathcal{P}$-linear coalgebra a cocommutative coalgebra in this monoidal category. One should not confuse the notion of a $\mathcal{P}$-linear coalgebra with the notion of a $\mathcal{P}$-coalgebra. Classically, linear duality over a ring makes algebras and coalgebras dual to each other (see [25]). We define a similar duality between $\mathcal{P}$-algebras and $\mathcal{P}$-linear coalgebras.

The third section of the paper is devoted to the proof of theorem 0.1. In summary, we apply the duality between $\mathcal{P}$-algebras and $\mathcal{P}$-linear coalgebras to reduce the statement of the theorem to the classical one. In fact, the duality between $\mathcal{P}$-algebras and $\mathcal{P}$-linear coalgebras converts a cogroup object into a group object. Furthermore, as for classical coalgebras, a group in the $\mathcal{P}$-linear coalgebras is nothing but a $\mathcal{P}$-linear Hopf algebra (a Hopf algebra in the monoidal category of right $\mathcal{P}$-modules). Now, algebras, coalgebras, or Hopf algebras in a monoidal category behave like the classical algebras, coalgebras or Hopf algebras.

The fourth section is concerned with theorem 0.3. By linear duality over the ground field, a $\mathcal{P}$-coalgebra is dual to a pseudocompact $\mathcal{P}$-algebra. In the case, $\mathcal{P}=\mathcal{C}$ om, this duality is known as the Cartier duality. This duality enables us to obtain theorem 0.3 as a consequence of the pseudocompact variant of theorem 0.1 .

In section 5, we show how theorems 0.2 and 0.3 apply in the examples mentioned above.

In the appendix, we show that the theory of Hopf algebras in the category of graded $\mathbf{Q}$-modules can be extended to any graded $\mathbf{Q}$-linear symmetric monoidal category.

\subsection{Notation and conventions.}

Throughout this paper we work over a fixed field $k$.

We denote the symmetric group by $S_{n}$. The letter $\tau$ denotes the transposition (1 2 2) $\in S_{2}$. Let $\sigma \in S_{r}$. If $i_{1}+\cdots+i_{r}=n$, then $\sigma\left(i_{1}, \ldots, i_{r}\right)$ is the block permutation of $S_{n}$, defined by the formula

$$
\begin{aligned}
\sigma\left(i_{1}, \ldots, i_{r}\right)\left(i_{\sigma(1)}+\cdots+i_{\sigma(k-1)}+l\right) & :=i_{1}+\cdots+i_{\sigma(k)-1}+l, \\
& \text { for } l=1, \ldots, i_{\sigma(k)} \text { and } k=1, \ldots, r .
\end{aligned}
$$

For the tranposition $\tau=\left(\begin{array}{ll}1 & 2\end{array}\right)$ introduced above, we have

$$
\begin{aligned}
& \tau(i, j)(l) \quad=i+l, \quad l=1, \ldots, j, \\
& \tau(i, j)(j+l)=l, \quad l=1, \ldots, i \text {. }
\end{aligned}
$$

In the sequel, many $k$-modules are endowed with a natural $S_{n}$-action. We denote this action by $\sigma \cdot v$, where $\sigma$ belongs to $S_{n}$ and $v$ belongs to an $S_{n}$-module $V$. Here is the main example where this convention is applied. Let $V$ be an $S_{i^{-}}$ module and $W$ be an $S_{j}$-module. Consider the induced module $\operatorname{Ind}_{S_{i} \times S_{j}}^{S_{n}} V \otimes W$. 
Let $v \in V, w \in W$. By an abuse of notation, we denote by $v \otimes w$ the element $1 \otimes v \otimes w \in k\left[S_{n}\right] \otimes_{k\left[S_{i} \times S_{j}\right]} V \otimes W=\operatorname{Ind}_{S_{i} \times S_{j}}^{S_{n}} V \otimes W$. Thus, if $\sigma \in S_{n}$, then $\sigma \cdot v \otimes w$ denotes the action of $\sigma$ on $v \otimes w$ in $\operatorname{Ind}_{S_{i} \times S_{j}}^{S_{n}} V \otimes W$, hence, the tensor $\sigma \otimes v \otimes w \in \operatorname{Ind}_{S_{i} \times S_{j}}^{S_{n}} V \otimes W$. Some modules are equipped with extra $S_{n}$-actions, which should not be confused with the natural action of the symmetric group (cf. 1.1.4).

\section{$\S 1$. Preliminaries}

\subsection{Operads and algebras}

This section provides a survival kit on operads and their algebras. A more complete exposition of the theory of operads may be found in [5], [6], [8], [12], [15], or [18].

1.1.1. Definition. An $\mathbf{S}$-module $V$ is a sequence of $S_{n}$-modules $V(n), n \in \mathbf{N}$. The S-modules form a graded abelian category (cf. A.1): if we consider the $S_{n}$-module $V(n)$ as an $\mathbf{S}$-module concentrated in degree $n$, then, in the category of $\mathbf{S}$-modules, we have the decomposition formula

$$
V=\oplus_{n \geq 0} V(n)
$$

We may call $V(n)$ the degree $n$ component of $V$. As for graded modules, the notation $v \in V(n)$ may be abbreviated to $v \in V$. The integer $n$ is called the degree of $v$ and we denote $|v|=n$.

\subsubsection{The functor associated to an S-module}

An $\mathbf{S}$-module $V$ gives rise to a functor $T(V,-)$ on the category of $k$-modules. This functor is defined by the formula

$$
T(V, X):=\bigoplus_{n=0}^{\infty} V(n) \otimes_{S_{n}} X^{\otimes n} .
$$

Moreover, this construction provides an embedding from the category of S-modules to the category of functors on $k$-modules. The composite of $T(V,-)$ and $T(W,-)$ is again given by an $\mathbf{S}$-module (cf. [8]). More precisely, there exists a bifunctor (called the composition product of $\mathbf{S}$-modules)

$$
\circ: \mathbf{S}-\operatorname{Mod} \times \mathbf{S}-\operatorname{Mod} \longrightarrow \text { S-Mod, }
$$

such that 


$$
T(V \circ W,-)=T(V, T(W,-)) .
$$

We give an explicit formula for the composition product of $\mathbf{S}$-modules in 1.1.4.

\subsubsection{The tensor product of S-modules}

There also exists a bifunctor (called the tensor product of S-modules)

$$
\otimes: \text { S-Mod } \times \text { S-Mod } \longrightarrow \text { S-Mod, }
$$

such that

$$
T(V \otimes W, X)=T(V, X) \otimes T(W, X) .
$$

In fact, it is easy to see that the $\mathbf{S}$-module

$$
(V \otimes W)(n)=\bigoplus_{i+j=n} \operatorname{Ind}_{S_{i} \times S_{j}}^{S_{n}} V(i) \otimes W(j)
$$

verifies the identity above. The tensor product of $\mathbf{S}$-modules is unital, associative and commutative, because the tensor product of functors is. Hence, the category of $\mathbf{S}$-modules is equipped with the structure of a symmetric monoidal category. Moreover, in some sense, the formula (1.1.3) above shows that the tensor product of $\mathbf{S}$-modules preserves the "total degree". To conclude, the $\mathbf{S}$-modules form a graded $k$-linear symmetric monoidal category (see A.1).

The unit of the tensor product of $\mathbf{S}$-modules is the $\mathbf{S}$-module 1, which is concentrated in degree 0 and has $1(0)=k$. It is easy to check directly on the formula (1.1.3) above that the tensor product of $\mathbf{S}$-modules is associative: clearly, the $n$-fold tensor product may be written

$$
\left(V_{1} \otimes \cdots \otimes V_{r}\right)(n)=\bigoplus_{i_{1}+\cdots+i_{r}=n} \operatorname{Ind}_{S_{i_{1}} \times \cdots \times S_{i_{r}}}^{S_{n}} V\left(i_{1}\right) \otimes \cdots \otimes V\left(i_{r}\right) .
$$

As explained in 0.5, the formula $\sigma \cdot v \otimes w$, where $\sigma \in S_{n}, v \in V(i)$, and $w \in W(j)$ denotes a tensor in $\operatorname{Ind}_{S_{i} \times S_{j}}^{S_{n}} V(i) \otimes W(j)$. The symmetry operator $\tau_{*}: V \otimes$ $W \stackrel{\simeq}{\longrightarrow} W \otimes V$ is given by

$$
\tau_{*}(\sigma \cdot v \otimes w)=\sigma \tau(i, j) \cdot w \otimes v .
$$

Recall that $\tau$ denotes the transposition $\left(\begin{array}{ll}1 & 2\end{array}\right) \in S_{2}$ and $\tau(i, j)$ is the associated block transposition of $S_{i+j}$ (cf. convention 0.5). 
1.1.4. The tensor power of an S-module and the composition product Let $W$ be an $\mathbf{S}$-module. Since the tensor product of $\mathbf{S}$-modules is symmetric, any $s \in S_{r}$ gives rise to a morphism of $\mathbf{S}$-modules

$$
s^{*}: W^{\otimes r} \longrightarrow W^{\otimes r} .
$$

Explicitly, if $w_{1} \in W\left(i_{1}\right), \ldots, w_{r} \in W\left(i_{r}\right)$, then

$$
s^{*}\left(\sigma \cdot w_{1} \otimes \cdots \otimes w_{r}\right):=\sigma \cdot s\left(i_{1}, \ldots, i_{r}\right) \cdot w_{s(1)} \otimes \cdots \otimes w_{s(r)} .
$$

We have clearly $(s t)^{*}=t^{*} s^{*}$ and it is natural to denote $s_{*}:=\left(s^{-1}\right)^{*}$. Thus, the tensor power $W^{\otimes r}$ is endowed with an $S_{r}$-action which is compatible with the $\mathrm{S}$-module structure. Let us mention that one should not confuse this $S_{r}$-action with the $\mathbf{S}$-module structure of $W^{\otimes r}$.

One may show that the $\mathbf{S}$-module

$$
(V \circ W)(n)=\bigoplus_{r=0}^{\infty}\left[V(r) \otimes\left(W^{\otimes r}(n)\right)\right]_{S_{r}}
$$

verifies the identity (1.1.1).

\subsubsection{Operads}

An operad is an $\mathbf{S}$-module $\mathcal{P}$, whose associated functor $T(\mathcal{P},-)$ is equipped with a monad structure (cf. [14]). An algebra over the $\operatorname{monad} T(\mathcal{P},-)$ is called a $\mathcal{P}$-algebra.

Of course, the monad product $\mu: T(\mathcal{P}, T(\mathcal{P},-)) \longrightarrow T(\mathcal{P},-)$ is equivalent to an $\mathbf{S}$-module morphism $\mu: \mathcal{P} \circ \mathcal{P} \longrightarrow \mathcal{P}$. Furthermore, an operad structure may be specified by products

$$
\mathcal{P}(r) \otimes \mathcal{P}\left(i_{1}\right) \otimes \cdots \otimes \mathcal{P}\left(i_{r}\right) \longrightarrow \mathcal{P}\left(i_{1}+\cdots+i_{r}\right),
$$

satisfying some natural conditions (cf. [18]). This follows from the formula (1.1.4) above. The image of $\mu \otimes \nu_{1} \otimes \cdots \otimes \nu_{r}$ under this map is usually denoted by $\mu\left(\nu_{1}, \ldots, \nu_{r}\right)$.

In the same way, a $\mathcal{P}$-algebra structure on a $k$-module $R$ is equivalent to a sequence of $S_{n}$-equivariant products

$$
\mathcal{P}(n) \otimes R^{\otimes n} \longrightarrow R
$$

which are associative with respect to the operad product. Let $\mu \in \mathcal{P}(n), r_{1}, \ldots r_{n} \in$ $R$. The image of $\mu \otimes r_{1} \otimes \cdots \otimes r_{n}$ under the product above is usually denoted by $\mu\left(r_{1}, \ldots, r_{n}\right)$. As for any monad, if $V$ is a $k$-module, then $T(\mathcal{P}, V)$ is the free 
$\mathcal{P}$-algebra generated by $V$.

1.1.6. Assumption. In the sequel, any operad $\mathcal{P}$ is assumed to be unital (explicitly, $\mathcal{P}(0)=0$ and $\mathcal{P}(1)=k)$.

1.1.7. Examples. As mentioned in the introduction, there are operads $\mathcal{C} o m, \mathcal{A} s$, $\mathcal{L} i e$, whose algebras are respectively, the commutative algebras (without unit), the associative algebras (without unit), and the Lie algebras. We just recall the expansion of the associated free algebra functors. The free commutative algebra is the symmetric algebra

$$
T(\mathcal{C o m}, V)=\bar{S}(V)=\bigoplus_{n=1}^{\infty}\left(V^{\otimes n}\right)_{S_{n}} .
$$

The free associative algebra is the tensor algebra

$$
T(\mathcal{A} s, V)=\bar{T}(V)=\bigoplus_{n=1}^{\infty} V^{\otimes n} .
$$

In characteristic zero, the free Lie algebra is the primitive part of the tensor algebra equipped with the "shuffle" coproduct

$$
T(\mathcal{L} i e, V)=\operatorname{Prim} \bar{T}(V)
$$

It can also be described as

$$
T(\mathcal{L} i e, V)=\bigoplus_{n=1}^{\infty} e_{n}^{[1]} V^{\otimes n},
$$

where $e_{n}^{[1]}$ is an idempotent of $k\left[S_{n}\right]$ known as the first Eulerian idempotent (cf. [22]).

1.1.8. Ideals. An ideal of a $\mathcal{P}$-algebra $R$ is a submodule of $R$, say $I$, such that

$$
\mu\left(r_{1}, \ldots, r_{n-1}, x\right) \in I, \forall \mu \in \mathcal{P}(n), \forall r_{1}, \ldots, r_{n-1} \in R, \forall x \in I .
$$

Clearly, the $\mathcal{P}$-algebra product $T(\mathcal{P}, R) \longrightarrow R$ induces a $\mathcal{P}$-algebra product on the quotient $R / I$.

Let us denote by $R^{(n)}$ the image of $\oplus_{i \geq n} \mathcal{P}(i) \otimes_{S_{i}} R^{\otimes i}$ under the $\mathcal{P}$-algebra product. Of course, $R^{(n)}$ is an ideal of $R$, called the $n$-th power of the augmentation ideal of $R$.

In the case $\mathcal{P}=\mathcal{C}$ om, $\mathcal{A} s$, we recover the classical notion of a (two-sided) ideal. 
In the case $\mathcal{P}=\mathcal{L} i e$, we recover the notion of a Lie ideal. Moreover, if $L$ is a Lie algebra, then $L^{(n)}, n \in \mathbf{N}$, is the lower central series of $L$.

A pair of parallel $\mathcal{P}$-algebra morphisms $d_{0}, d_{1}: A \longrightarrow B$ is reflexive, when there exists a linear map $s_{0}: B \longrightarrow A$, such that $d_{0} s_{0}=d_{1} s_{0}=B$. The coequalizer of a reflexive pair is known as a reflexive coequalizer.

1.1.9. Lemma. The forgetful functor from the $\mathcal{P}$-algebras to the $k$-modules creates the reflexive coequalizers.

Proof. Let $d_{0}, d_{1}, s_{0}$ be as above. We show that the image of $d_{0}-d_{1}$ is an ideal of $B$. As a consequence, the $\mathcal{P}$-algebra product $T(\mathcal{P}, A) \longrightarrow A$ induces a $\mathcal{P}$-algebra product on the difference cokernel coker $\left(d_{0}-d_{1}\right)$, and this proves the lemma.

Let $b_{1}, \ldots, b_{n-1} \in B, a \in A, \mu \in \mathcal{P}(n)$. We have

$$
\begin{aligned}
& \mu\left(b_{1}, \ldots, b_{n-1}, d_{0} a\right) \\
= & \mu\left(d_{0} s_{0} b_{1}, \ldots, d_{0} s_{0} b_{n-1}, d_{0} a\right) \\
= & d_{0} \mu\left(s_{0} b_{1}, \ldots, s_{0} b_{n-1}, a\right) \\
= & \left(d_{0}-d_{1}\right) \mu\left(s_{0} b_{1}, \ldots, s_{0} b_{n-1}, a\right)+d_{1} \mu\left(s_{0} b_{1}, \ldots, s_{0} b_{n-1}, a\right) \\
= & \left(d_{0}-d_{1}\right) \mu\left(s_{0} b_{1}, \ldots, s_{0} b_{n-1}, a\right)+\mu\left(b_{1}, \ldots, b_{n-1}, d_{1} a\right) .
\end{aligned}
$$

The category of $\mathcal{P}$-algebras is complete and cocomplete (see [5]). In particular, it possesses a coproduct which is denoted by $R \vee S$. The next lemma provides a tractable realization of $R \vee S$. Moreover, its demonstration gives also an alternative proof of the existence of $R \vee S$. Let $X$ be a $\mathcal{P}$-algebra. We denote the $\mathcal{P}$-algebra product by $\lambda_{X}: T(\mathcal{P}, X) \longrightarrow X$.

1.1.10. Lemma. Let $R, S$ be $\mathcal{P}$-algebras. Let $d_{0}=T\left(\mathcal{P}, \lambda_{R} \oplus \lambda_{S}\right)$. Let $d_{1}$ be the composite

$$
T(\mathcal{P}, T(\mathcal{P}, R) \oplus T(\mathcal{P}, S)) \hookrightarrow T(\mathcal{P}, T(\mathcal{P}, R \oplus S)) \stackrel{\mu_{R \oplus S}}{\longrightarrow} T(\mathcal{P}, R \oplus S),
$$

where $\mu_{R \oplus S}$ denotes the monad product. We have $R \vee S=\operatorname{coker}\left(d_{0}-d_{1}\right)$. Moreover, the $\mathcal{P}$-algebra product $T(\mathcal{P}, T(\mathcal{P}, R \oplus S)) \longrightarrow T(\mathcal{P}, R \oplus S)$ induces a $\mathcal{P}$ algebra product on the quotient $\operatorname{coker}\left(d_{0}-d_{1}\right)$, which coincides with the $\mathcal{P}$-algebra product of $R \vee S$.

Proof. Let $s_{0}=T\left(\mathcal{P}, \eta_{R} \oplus \eta_{S}\right)$, where $\eta_{X}: X \longrightarrow T(\mathcal{P}, X)$ denotes the monad unit. We have $d_{0} s_{0}=d_{1} s_{0}=1$. By the previous lemma, the $\mathcal{P}$-algebra product $T(\mathcal{P}, T(\mathcal{P}, R \oplus S)) \longrightarrow T(\mathcal{P}, R \oplus S)$ induces a $\mathcal{P}$-algebra product on the difference 
cokernel

$$
T\left(\mathcal{P}, \operatorname{coker}\left(d_{0}-d_{1}\right)\right) \longrightarrow \operatorname{coker}\left(d_{0}-d_{1}\right)
$$

Hence, $\operatorname{coker}\left(d_{0}-d_{1}\right)$ is a $\mathcal{P}$-algebra and the $k$-linear coequalizer

$$
T(\mathcal{P}, T(\mathcal{P}, R) \oplus T(\mathcal{P}, S)) \underset{d_{1}}{\stackrel{d_{0}}{\longrightarrow}} T(\mathcal{P}, R \oplus S) \longrightarrow \operatorname{coker}\left(d_{0}-d_{1}\right),
$$

is in fact a $\mathcal{P}$-algebra coequalizer.

Let $X$ be a $\mathcal{P}$-algebra. A pair of $k$-linear maps $(R \stackrel{u}{\longrightarrow} X, S \stackrel{v}{\longrightarrow} X)$ induces a $\mathcal{P}$-algebra morphism $T(\mathcal{P}, R \oplus S) \longrightarrow X$. It is not hard to see that this arrow factors through the coequalizer if and only if $u$ and $v$ are $\mathcal{P}$-algebra morphisms. As a consequence, $R \vee S=\operatorname{coker}\left(d_{0}-d_{1}\right)$.

1.1.11. Complete algebras. A $\mathcal{P}$-algebra is said to be nilpotent if the product $\mathcal{P}(n) \otimes R^{\otimes n} \longrightarrow R$ vanishes for $n$ large. A complete (or pronilpotent) $\mathcal{P}$-algebra is a $\mathcal{P}$-algebra $R$ together with a sequence of ideals $I_{n}, n \geq 1$, such that the $\mathcal{P}$-algebra $R / I_{n}$ is nilpotent and $R=\lim R / I_{n}$. Notice that the hypothesis $R / I_{n}$ nilpotent implies that the topology defined by the $n$-th powers of the augmentation ideal $R^{(n)}$ is equivalent to the topology defined by the $I_{n}$ 's. As a consequence, any $\mathcal{P}$-algebra morphism is continuous. In the cases $\mathcal{P}=\mathcal{C}$ om, $\mathcal{A} s, \mathcal{L} i e$, we recover the classical notions of a (pro)nilpotent commutative algebra, of a (pro)nilpotent associative algebra, and of a (pro)nilpotent Lie algebra.

We denote by $\widehat{\mathcal{P}}(V)$ the free complete $\mathcal{P}$-algebra generated by a $k$-module $V$. Explicitly, $\widehat{\mathcal{P}}(V)$ is the completion of the free $\mathcal{P}$-algebra generated by $V$ with respect to the powers of the augmentation ideal $I_{n}:=\oplus_{i \geq n} \mathcal{P}(i) \otimes_{S_{i}} V^{\otimes i}$. Thus, we have

$$
\widehat{\mathcal{P}}(V)=\prod_{i=1}^{\infty} \mathcal{P}(i) \otimes_{S_{i}} V^{\otimes i} .
$$

In the sequel, we denote an homogeneous element of $\widehat{\mathcal{P}}(V)$ by $\mu\left(v_{1}, \ldots, v_{n}\right)$ instead of $\mu \otimes v_{1} \otimes \cdots \otimes v_{n}$.

We denote by $\widehat{\mathcal{P}}\left(x_{1}, \ldots, x_{n}\right)$ the free complete $\mathcal{P}$-algebra generated by $n$ variables $x_{1}, \ldots, x_{n}$. By definition, we have $\widehat{\mathcal{P}}\left(x_{1}, \ldots, x_{n}\right)=\widehat{\mathcal{P}}(V)$, where $V=$ $\operatorname{Span}\left\langle x_{1}, \ldots, x_{n}\right\rangle$. An element of $\widehat{\mathcal{P}}\left(x_{1}, \ldots, x_{n}\right)$ is also known as a $\mathcal{P}$-power series. If $\mathcal{P}$ is the commutative operad $\mathcal{C}$ om, then $\widehat{\mathcal{C o m}}\left(x_{1}, \ldots, x_{n}\right)$ is the classical power series algebra (or more exactly its augmentation ideal). If $\mathcal{P}$ is the associative op$\operatorname{erad} \mathcal{A} s$, then $\widehat{\mathcal{A s}}\left(x_{1}, \ldots, x_{n}\right)$ is the algebra of power series in $n$ non-commutative variables. A power series over the Lie operad is a formal sum of Lie polynomials.

The category of complete $\mathcal{P}$-algebras is equipped with a coproduct. Let $R=$ $\lim R / I_{n}, S=\lim S / J_{n}$ be complete $\mathcal{P}$-algebras. The coproduct of $R$ and $S$ is the 
$\mathcal{P}$-algebra $\lim R \vee S /\left(I_{n} \vee S+R \vee J_{n}\right)$ (in fact, this algebra is the completion of $R \vee S$ with respect to the sequence of ideals $I_{n} \vee S+R \vee J_{n}$ ). In the sequel, if $R$ and $S$ are supposed to be complete, then the notation $R \vee S$ denotes the completed coproduct. We denote by $\nabla: R \vee R \longrightarrow R$ the folding map, whose restriction to each summand of the coproduct is the identity.

The null space is a zero object in the category of complete $\mathcal{P}$-algebras. We denote by 0 the zero arrow.

A general notion of a power series, which contains the case of $\mathcal{P}$-power series, was first given by Lazard in [10], within the language of analyzers. In fact, the sequence of $\mathcal{P}$-algebras $\widehat{\mathcal{P}}\left(x_{1}, \ldots, x_{n}\right)$ form an analyzer. The interest in formal geometry over an operad starts with Kontsevich's paper [9]. The notion of a $\mathcal{P}$-power series was introduced in $[6]$.

1.1.12. Complete graded algebras. The general theory of operads can be handled in any symmetric monoidal category equipped with limits and colimits, allowing one to define graded or supergraded operads. For simplicity, we work with $k$-modules. Netherveless, it is straightforward to rewrite this paper in the graded context. In particular, theorem 0.1 remains valid for complete graded $\mathcal{P}$-algebras.

Let us explain why a connected graded $\mathcal{P}$-algebra $R$ turns out to be a complete graded $\mathcal{P}$-algebra. Recall that a graded object $V$ is called connected when $V_{d}=0$ for $d \leq 0$. Equip the graded $\mathcal{P}$-algebra $R$ with the topology defined by the sequence of homogeneous ideals $I^{(n)}$, whose degree $d$ component is given by

$$
I_{d}^{(n)}=\begin{array}{cl}
0, & \text { if } d<n, \\
R_{d}, & \text { otherwise. }
\end{array}
$$

Since $R$ is connected, the $\mathcal{P}$-algebra $R / I^{(n)}$ is nilpotent. Moreover, $R_{d}=R_{d} / I_{d}^{(n)}$ for $n$ large. Thus, we have $R=\lim R / I^{(n)}$, where lim denotes the limit in the category of graded $n$-modules. Hence, $R$ is a complete graded $\mathcal{P}$-algebra. Therefore, theorem 0.2 is a particular instance of theorem 0.1 .

\subsection{Cogroups in algebras over an operad}

1.2.1. Definition. A cogroup in the category of complete $\mathcal{P}$-algebras is a complete $\mathcal{P}$-algebra $R$ together with a "coproduct" $\gamma: R \longrightarrow R \vee R$, and an "antipode" $\iota: R \longrightarrow R$ which verify the following classical identities:

$$
\begin{aligned}
\gamma \vee R \cdot \gamma & =R \vee \gamma \cdot \gamma, \\
R \vee 0 \cdot \gamma & =0 \vee R \cdot \gamma=R, \\
\nabla \cdot R \vee \iota \cdot \gamma & =\nabla \cdot \iota \vee R \cdot \gamma=0
\end{aligned}
$$


Actually, we do not need to require the existence of the antipode $\iota$ in the definition, because the next lemma ensures that the antipode always exists.

1.2.2. Lemma. Let $R$ be a $\mathcal{P}$-algebra equipped with a coproduct $\gamma: R \longrightarrow R \vee R$ satisfying equations (1.2.1) and (1.2.2). There exists a unique map $\iota: R \longrightarrow R$ which is a solution to the equation (1.2.3).

Proof.

1) We construct the map $\iota$ by a step by step approximation process. As pointed out before (cf. 1.1.11), we can assume that the topology on $R$ is given by the powers of the augmentation ideal. Assume that we have built a sequence of coherent maps $\iota_{n}: R \longrightarrow R / R^{(n)}$ such that the composite

$$
R \stackrel{\gamma}{\longrightarrow} R \vee R \stackrel{R \vee \iota_{n}}{\longrightarrow} R \vee R / R^{(n)} \stackrel{\nabla}{\longrightarrow} R / R^{(n)}
$$

is the zero morphism. The word coherent means that $\iota_{n+1} \equiv \iota_{n}\left(\bmod R^{(n)}\right)$.

By completeness, there exists a map $\iota$ such that $\iota \equiv \iota_{n}\left(\bmod R^{(n)}\right)$. Moreover, $\iota$ is a solution to the equation $\nabla \cdot R \vee \iota \cdot \gamma=0$. In the same way, we can construct a map $\iota^{\prime}$ such that $\nabla \cdot \iota^{\prime} \vee R \cdot \gamma=R$. Let us denote by $\nabla_{3}$ the map defined by

$$
\nabla_{3}=\nabla \cdot \nabla \vee R=\nabla \cdot R \vee \nabla
$$

In the same way, we denote by $\gamma_{3}$ the 3 -fold coproduct of $R$. We have

$$
\iota=\nabla \cdot 0 \vee \iota \cdot \gamma=\nabla_{3} \cdot \iota^{\prime} \vee R \vee \iota \cdot \gamma_{3}=\nabla \cdot \iota^{\prime} \vee 0 \cdot \gamma=\iota^{\prime}
$$

Hence, $\iota$ is a two sided inverse. The same argument proves the uniqueness of $\iota$.

2) We construct the maps $\iota_{n}$ by induction on $n$. First, we decompose the coproduct of two $\mathcal{P}$-algebras into a quadratic part and a linear part. Let $R, S$ be $\mathcal{P}$-algebras. Let us denote by $i, j$ the canonical morphisms

$$
R \stackrel{i}{\longrightarrow} R \vee S \stackrel{j}{\longleftarrow} S .
$$

There are also canonical projections

$$
R \stackrel{p}{\longleftarrow} R \vee S \stackrel{q}{\longrightarrow} S .
$$

where $p$ is characterized by $p i=R, p j=0$, and $q$ by $q i=0, q j=S$. Thus, the $k$-linear map $i+j: R \oplus S \longrightarrow R \vee S$ is a continuous section of the morphism $(p, q): R \vee S \longrightarrow R \oplus S$. (Warning: $i+j$ is not a $\mathcal{P}$-algebra morphism). Thus, we have a natural exact sequence

$$
0 \longrightarrow(R, S) \longrightarrow R \vee S \stackrel{(p, q)}{\longrightarrow} R \oplus S \longrightarrow 0,
$$


which possesses a continuous linear splitting. The kernel of $(p, q)$, denoted by $(R, S)$ is known as the cross effect of the coproduct.

In the same way, we define $T(\mathcal{P} ; R, S)$, the cross effect of the functor $T(\mathcal{P},-)$, as the kernel of the canonical projection $T(\mathcal{P}, R \oplus S) \longrightarrow T(\mathcal{P}, R) \oplus T(\mathcal{P}, S)$. This projection has a section given by the canonical inclusions. We have a morphism of split exact sequences given by the diagram

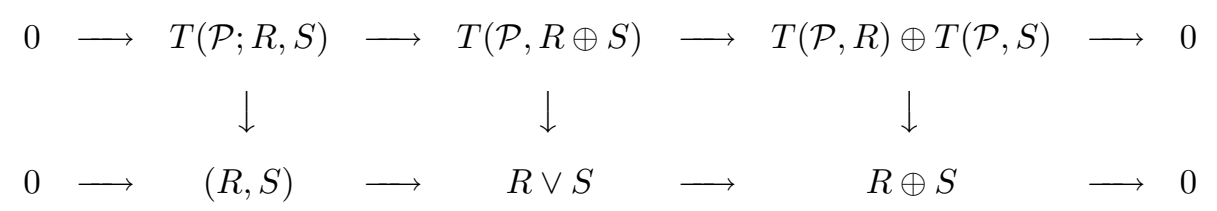

In this diagram, the right vertical arrow is induced by the $\mathcal{P}$-algebra product of $R$ and $S$. The middle vertical arrow is the canonical map $T(\mathcal{P}, R \oplus S) \longrightarrow R \vee S$. One sees immediately that the right square of the diagram commutes. It is also easy to check that the splittings are compatible. As a consequence, the image of the left vertical arrow is dense into $(R, S)$.

The restriction of the folding map to the cross effect

$$
\left(R, R / R^{(n+1)}\right) \stackrel{\nabla}{\longrightarrow} R / R^{(n+1)}
$$

vanishes over the image of $T\left(\mathcal{P} ; R, R^{(n)} / R^{(n+1)}\right)$ in $\left(R, R / R^{(n+1)}\right)$. Therefore, this restriction factors through the canonical projection $\left(R, R / R^{(n+1)}\right) \longrightarrow\left(R, R / R^{(n)}\right)$. We denote this factorisation by $\bar{\nabla}$.

3) Let us show the uniqueness of $\iota_{n+1}$. Let $Q_{n}$, resp. $Q_{n+1}$, be the projection of $\iota_{n}$, resp. $\iota_{n+1}$, onto the summand $\left(R, R / R^{(n)}\right)$, resp. $\left(R, R / R^{(n+1)}\right)$. By naturality of this projection, the composite of $Q_{n+1}$ with the canonical map $\left(R, R / R^{(n+1)}\right) \longrightarrow\left(R, R / R^{(n)}\right)$ is equal to $Q_{n}$. Thus, we have $\nabla Q_{n+1}=\bar{\nabla} Q_{n}$. Equation (1.2.2) implies that the projection of $\gamma$ onto the summand $R \oplus R$ of $R \vee R / R^{(n+1)}$ is equal to $\left(R, \iota_{n+1}\right)$.

Finally, equation (1.2.4) is equivalent to

$$
r+\iota_{n+1}(r)+\bar{\nabla} Q_{n}(r)=0, \forall r \in R
$$

This equation proves the uniqueness of $\iota_{n+1}$. 
It remains to show the existence of $\iota_{n+1}$. More precisely, we show that the map $\iota_{n+1}$ defined by equation (1.2.5) is a $\mathcal{P}$-algebra morphism. Let $\mu \in \mathcal{P}(i)$. For simplicity, we assume $i=2$. By definition of $Q_{n}$, we have in $R \vee R / R^{(n)}$

$$
\begin{aligned}
\mu\left(r+\iota_{n}(r)+Q_{n}(r), s+\iota_{n}(s)+Q_{n}(s)\right)=\mu\left(R \vee \iota_{n} \cdot \gamma(r), R \vee \iota_{n} \cdot \gamma(s)\right) \\
=R \vee \iota_{n} \cdot \gamma(\mu(r, s))=\mu(r, s)+\iota_{n}(\mu(r, s))+Q_{n}(\mu(r, s)) .
\end{aligned}
$$

Since $\iota_{n}$ is a $\mathcal{P}$-algebra morphism, we obtain the equation in $\left(R, R / R^{(n)}\right)$

$Q_{n}(\mu(r, s))=\mu\left(\iota_{n} r, s\right)+\mu\left(r, \iota_{n} s\right)+\mu\left(r+\iota_{n} r, Q_{n} s\right)+\mu\left(Q_{n} r, s+\iota_{n} s\right)+\mu\left(Q_{n} r, Q_{n} s\right)$.

Using this equation, we obtain

$$
\begin{aligned}
0 & =\mu\left(r+\iota_{n+1} r+\bar{\nabla} Q_{n}(r), s+\iota_{n+1} s+\bar{\nabla} Q_{n}(s)\right) \\
& =\mu\left(\iota_{n+1} r, \iota_{n+1} s\right)+\mu(r, s)+\bar{\nabla} Q_{n}(\mu(r, s)) .
\end{aligned}
$$

By uniqueness, this implies $\iota_{n+1}(\mu(r, s))=\mu\left(\iota_{n+1} r, \iota_{n+1} s\right)$. Thus, we are done.

\subsubsection{Formal group laws}

Assume that $R$ is the free $\mathcal{P}$-algebra $\widehat{\mathcal{P}}\left(x_{1}, \ldots, x_{n}\right)$. In this case, the coproduct $\gamma: R \longrightarrow R \vee R$ is equivalent to a $\mathcal{P}$-formal group law. A $\mathcal{P}$-formal group law is an $n$-tuple of $\mathcal{P}$-power series

$$
G_{1}(x, y), \ldots, G_{n}(x, y) \in \widehat{\mathcal{P}}\left(x_{1}, \ldots, x_{n}, y_{1}, \ldots, y_{n}\right)
$$

which verify the identities

$$
\begin{gathered}
G_{i}(x, 0)=G_{i}(0, x)=0, \\
G_{i}(G(x, y), z)=G_{i}(x, G(y, z)) .
\end{gathered}
$$

(It is not hard to extend the composition of commutative power series to power series over an operad.) In fact, we have $R \vee R=\widehat{\mathcal{P}}\left(x_{1}, \ldots, x_{n}, y_{1}, \ldots, y_{n}\right)$, and the equivalence between the structures follows from the definition of the free complete $\mathcal{P}$-algebra. If $\mathcal{P}$ is the commutative operad, then we recover the classical notion of a formal group law.

The generalization of the notion of a formal group law to algebra structures, which are different from the commutative one, was first given by Lazard in the language of analyzers (cf. [10]). In this context, Lazard establishes a kind of Lie theory. Lazard theory was translated to the context of algebras over an operad by V. Ginzburg and M. Kapranov (cf. [6]). A more conceptual approach to Lazard Lie theory avoiding power series calculations is given in [4]. 
As in the case of commutative algebras, the Campbell-Hausdorff power series give rise to cogroups in the category of $\mathcal{P}$-algebras (see $[9$, p. 186]).

\section{$\S 2$. On $\mathcal{P}$-linear coalgebras and $\mathcal{P}$-algebras}

2.1. Definition. Let $(T, \mu: T T \rightarrow T, \eta: 1 \rightarrow T)$ be a monad. A right $T$-functor is a functor $M: \operatorname{Mod}_{k} \longrightarrow \operatorname{Mod}_{k}$ equipped with a right $T$ action, which is a natural transformation $\rho_{X}: M T(X) \longrightarrow M(X)$ such that

$$
\begin{aligned}
& \rho_{X} \cdot M\left(\mu_{X}\right)=\rho_{X} \cdot \rho_{T(X)}, \\
& \rho_{X} \cdot M\left(\eta_{X}\right)=X .
\end{aligned}
$$

A right $\mathcal{P}$-module is an $\mathbf{S}$-module $V$, whose associated functor $T(V, \mathcal{P})$ is equipped with the structure of a right $T(\mathcal{P},-)$-functor. As for operad, a right $\mathcal{P}$-module structure is given by an S-module morphism $V \circ \mathcal{P} \longrightarrow V$, which is associative and unital with respect to the operad product. Furthermore, the right $\mathcal{P}$-action is equivalent to a sequence of products

$$
V(n) \otimes \mathcal{P}\left(i_{1}\right) \otimes \cdots \otimes \mathcal{P}\left(i_{n}\right) \longrightarrow V\left(i_{1}+\cdots+i_{n}\right),
$$

which satisfy the May axioms, except that we put $V$ in the first position instead of $\mathcal{P}$. We denote by $v\left(\mu_{1}, \ldots, \mu_{n}\right)$ the action of $\mu_{1} \otimes \cdots \otimes \mu_{n}$ on $v \in V(n)$.

A morphism in the category of right $\mathcal{P}$-modules, say $\phi$, is also called a $\mathcal{P}$-linear map. This means that $\phi$ is linear with respect to the action of the operad $\mathcal{P}$. Explicitly, we have

$$
\phi\left(v\left(\mu_{1}, \ldots, \mu_{n}\right)\right)=\phi(v)\left(\mu_{1}, \ldots, \mu_{n}\right), \forall v \in V, \forall \mu_{1}, \ldots, \mu_{n} \in \mathcal{P} .
$$

We denote by $\operatorname{Mod}_{\mathcal{P}}$ the category of right $\mathcal{P}$-modules.

Right $\mathcal{P}$-modules are also considered by M. Markl in [15], [16], [17]. There is also a notion of a left $\mathcal{P}$-module and of a $\mathcal{P}$-bimodule (cf. [15, Definition 1.3]). The reference [16] provides nice geometrical examples of right modules over an operad.

\subsection{The tensor product of right $\mathcal{P}$-modules}

Recall that the tensor product of two $\mathbf{S}$-modules $V$ and $W$ is defined by the formula

$$
(V \otimes W)(n)=\bigoplus_{p+q=n} \operatorname{Ind}_{S_{p} \times S_{q}}^{S_{n}} V(p) \otimes W(q)
$$


and is characterized by the equation

$$
T(V \otimes W, X)=T(V, X) \otimes T(W, X) .
$$

We show that the tensor product of right $\mathcal{P}$-modules $V$ and $W$ is endowed with a canonical right $\mathcal{P}$-action. Hence, as for $\mathbf{S}$-modules, the right $\mathcal{P}$-modules form a graded $k$-linear symmetric monoidal category. Let $V, W$ be right $\mathcal{P}$-modules. Equivalently, $T(V,-)$ and $T(W,-)$ are right $T(\mathcal{P},-)$-functors. The canonical map

$T(V \otimes W, T(\mathcal{P}, X))=$

$$
=T(V, T(\mathcal{P}, X)) \otimes T(W, T(\mathcal{P}, X)) \longrightarrow T(V, X) \otimes T(W, X)=T(V \otimes W, X)
$$

provides $T(V \otimes W,-)$ with the structure of a right $T(\mathcal{P},-)$-functor. The right $\mathcal{P}$-action on $V \otimes W$ may also be given by the formula

$$
\begin{aligned}
(\sigma \cdot v \otimes w) & \left(\mu_{1}, \ldots, \mu_{n}\right) \\
& :=\sigma\left(i_{1}, \ldots, i_{n}\right) \cdot v\left(\mu_{\sigma(1)}, \ldots, \mu_{\sigma(p)}\right) \otimes w\left(\mu_{\sigma(p+1)}, \ldots, \mu_{\sigma(p+q)}\right)
\end{aligned}
$$

for $\sigma \cdot v \otimes w \in \operatorname{Ind}_{S_{p} \times S_{q}}^{S_{n}} V(p) \otimes W(q)$ and $\mu_{1} \in \mathcal{P}\left(i_{1}\right), \ldots, \mu_{n} \in \mathcal{P}\left(i_{n}\right)$.

\subsection{On $\mathcal{P}$-linear coalgebras}

We call $\mathcal{P}$-linear coalgebra a coassociative coalgebra in the symmetric monoidal category of right $\mathcal{P}$-modules (cf. A.2). Explicitly, a $\mathcal{P}$-linear coalgebra is a right $\mathcal{P}$-module $C$ equipped with a coproduct, which is a $\mathcal{P}$-linear map $\Delta: C \longrightarrow C \otimes C$. By definition of the tensor product $C \otimes C$, the coproduct of an element $c \in C(n)$ has an expansion of the form

$$
\Delta c=\sum_{(c)} \sigma^{i} \cdot c_{(1)}^{i} \otimes c_{(2)}^{i}
$$

where $\sigma^{i} \in S_{n}, c_{(1)}^{i} \in C(p), c_{(2)}^{i} \in C(q)$ and $p+q=n$. The right-hand side of this formula is the classical Sweedler notation for a coproduct in the context of right $\mathcal{P}$-modules. More generally, given $c \in C$, the expression $\sum_{(c)} \sigma^{i} \cdot c_{(1)}^{i} \otimes \cdots \otimes c_{(n)}^{i}$ denotes the $n$-fold coproduct of $c$. We may omit the summation index $i$.

In the sequel, any $\mathcal{P}$-linear coalgebra is tacitely assumed to be cocommutative and connected. With the notation above, the cocommutativity of the coproduct may be written

$$
\sum_{(c)} \sigma \cdot c_{(1)} \otimes c_{(2)}=\sum_{(c)} \sigma \tau\left(\left|c_{(1)}\right|,\left|c_{(2)}\right|\right) \cdot c_{(2)} \otimes c_{(1)} .
$$


In this equation, $\left|c_{(1)}\right|$, resp. $\left|c_{(2)}\right|$, denotes the degree of $c_{(1)}$, resp. $c_{(2)}$ (see 1.1.1). In this context, the assumption "the coalgebra $C$ is connected" means $C(0)=k$ and $\Delta(1)=1 \otimes 1$.

A right $\mathcal{P}$-module $V$ is said to be connected when $V(0)=0$. In this case, the cofree cocommutative coalgebra generated by $V$ is the $\mathbf{S}$-module

$$
C(V)=\oplus_{n \geq 0}\left(V^{\otimes n}\right)^{S_{n}}
$$

equipped with the deconcatenation coproduct (cf. A.3). This assertion is a particular instance of proposition A.4.

In the appendix, we survey the theory of coalgebras and Hopf algebras in the general context of a graded $k$-linear symmetric monoidal category. In the next paragraph, we introduce a notion which is specific to the coalgebras in the monoidal category of right $\mathcal{P}$-modules.

\subsection{On $\mathcal{P}$-linear coalgebra $/ \mathcal{P}$-algebra pairings}

Let $C$ be a $\mathcal{P}$-linear coalgebra. Let $R$ be a complete $\mathcal{P}$-algebra. We endow the S-module $(C \otimes R)(n):=C(n) \otimes R$ with the obvious right $\mathcal{P}$-action. A $\mathcal{P}$-linear coalgebra/P-algebra pairing between $C$ and $R$ is a $\mathcal{P}$-linear map

$$
\Phi: C \otimes R \longrightarrow \mathcal{P}
$$

which makes the coproduct of $C$ adjoint to the product of $R$. Explicitly, we have the relation

$$
\Phi\left(c, \mu\left(r_{1}, \ldots, r_{n}\right)\right)=\sum_{(c)} \sigma^{i} \cdot \mu\left(\Phi\left(c_{(1)}^{i}, r_{1}\right), \ldots, \Phi\left(c_{(n)}^{i}, r_{n}\right)\right),
$$

for all $\mu \in \mathcal{P}(n), c \in C$, and $r_{1}, \ldots, r_{n} \in R$. Recall that the tensor $\sum_{(c)} \sigma^{i} \cdot c_{(1)}^{i} \otimes$ $\cdots \otimes c_{(n)}^{i}$ denotes the $n$-fold coproduct of $c$ (cf. 2.3). Notice that since $\mathcal{P}(0)=0$, we have $\Phi(1, r)=0$.

We denote by $\mathcal{B i l}_{\mathcal{P}}(C, R)$ the $k$-module of $\mathcal{P}$-linear coalgebra $/ \mathcal{P}$-algebra pairings between $C$ and $R$.

\subsection{A canonical pairing}

Let $V$ be a $k$-module. We denote by $\operatorname{Mod}_{k}(V, \mathcal{P})$ the right $\mathcal{P}$-module, whose degree $n$ component is $\operatorname{Mod}_{k}(V, \mathcal{P}(n))$, equipped with the obvious right $\mathcal{P}$-action. Let $f \in \operatorname{Mod}_{k}(V, \mathcal{P}(n)), v \in V$. We denote by $\langle f, v\rangle$ the image of $v$ under $f$. The action of $\mu_{1}, \ldots, \mu_{n} \in \mathcal{P}$ on $f$ may be written

$$
\left\langle f\left(\mu_{1}, \ldots, \mu_{n}\right), v\right\rangle=\langle f, v\rangle\left(\mu_{1}, \ldots, \mu_{1}\right) .
$$


Recall that $C\left(\operatorname{Mod}_{k}(V, \mathcal{P})\right)$ denotes the cofree $\mathcal{P}$-linear coalgebra generated by the right $\mathcal{P}$-module $\operatorname{Mod}_{k}(V, \mathcal{P})$. We define a $\mathcal{P}$-linear coalgebra $/ \mathcal{P}$-algebra pairing

$$
\langle-,-\rangle: C\left(\operatorname{Mod}_{k}(V, \mathcal{P})\right) \otimes \widehat{\mathcal{P}}(V) \longrightarrow \mathcal{P}
$$

as follows. Let $c$ be any homogeneous element of order $n$ of $C\left(\operatorname{Mod}_{k}(V, \mathcal{P})\right)$. Hence, $c=\sum \sigma \cdot f_{1} \otimes \cdots \otimes f_{n}$, with $f_{1}, \ldots, f_{n} \in \operatorname{Mod}_{k}(V, \mathcal{P})$. Let $r=\mu\left(v_{1}, \ldots, v_{m}\right) \in$ $\widehat{\mathcal{P}}(V)$. We set

$$
\langle c, r\rangle= \begin{cases}\sum \sigma \cdot \mu\left(\left\langle f_{1}, v_{1}\right\rangle, \ldots,\left\langle f_{n}, v_{n}\right\rangle\right), & \text { if } n=m \\ 0, & \text { otherwise }\end{cases}
$$

This pairing is well defined since $\sum \sigma \cdot f_{1} \otimes \cdots \otimes f_{n}$ is invariant under the action of $S_{n}$. We let the reader check that it makes the coproduct of $C\left(\operatorname{Mod}_{k}(V, \mathcal{P})\right)$ adjoint to the product of $\widehat{\mathcal{P}}(V)$.

2.6. Theorem. We denote by $\widehat{\mathcal{P}}-$ Alg the category of complete $\mathcal{P}$-algebras and by $\operatorname{coAlg}\left(\operatorname{Mod}_{\mathcal{P}}\right)$ the category of connected $\mathcal{P}$-linear cocommutative coalgebras. There exists a pair of contravariant adjoint functors

$$
(-)^{\circ}: \widehat{\mathcal{P}}-A l g \rightleftarrows \operatorname{coAlg}\left(\operatorname{Mod}_{\mathcal{P}}\right):(-)^{*},
$$

which both represent the bifunctor $\mathcal{B i l}_{\mathcal{P}}(C, R)$. More precisely, we have canonical isomorphisms

$$
\widehat{\mathcal{P}}-\operatorname{Alg}\left(R, C^{*}\right) \simeq \mathcal{B} i l_{\mathcal{P}}(C, R) \simeq \operatorname{coAlg}\left(\operatorname{Mod}_{\mathcal{P}}\right)\left(C, R^{\circ}\right) .
$$

This adjunction is similar to the classical adjunction between algebras and coalgebras over a field (see [25]).

Proof.

1) The dual $\mathcal{P}$-algebra of a $\mathcal{P}$-linear coalgebra

Fix a $\mathcal{P}$-linear coalgebra $C$. Let $R$ be the $\mathcal{P}$-linear dual of $C$. Explicitly, $R$ is the $k$-module of $\mathcal{P}$-linear maps from $C$ to $\mathcal{P}$. We equip $R$ with the $\mathcal{P}$-algebra structure given by

$\mu\left(f_{1}, \ldots, f_{n}\right)(c):=\sum_{(c)} \sigma \cdot \mu\left(f_{1}\left(c_{(1)}\right), \ldots, f_{n}\left(c_{(n)}\right)\right), \forall f_{1}, \ldots, f_{n}: R \longrightarrow \mathcal{P}, \forall c \in C$

Let $I_{n}$ be the submodule of $R$ formed by the maps which vanish in degree less 
than $n$. Explicitly, a $\mathcal{P}$-linear map $f: C \longrightarrow \mathcal{P}$ belongs to $I_{n}$, if we have

$$
f(c)=0, \forall c \in C(i) \text {, with } i<n .
$$

The $k$-module $I_{n}$ is clearly an ideal of $R$. Moreover $R / I_{n}$ is a nilpotent $\mathcal{P}$-algebra, and it is not hard to check that $R=\lim R / I_{n}$.

Let $S$ be a $\mathcal{P}$-algebra. There is an obvious one-to-one correspondence between $k$-linear maps from $S$ to $R$ and $\mathcal{P}$-linear maps from $C \otimes S$ to $\mathcal{P}$. Moreover $\mathcal{P}$ algebra morphisms and $\mathcal{P}$-linear coalgebra $/ \mathcal{P}$-algebra pairings correspond to each other under this correspondence. Hence, we have the identity

$$
\widehat{\mathcal{P}}-A l g(S, R) \simeq \mathcal{B} i l_{\mathcal{P}}(C, S)
$$

and $C^{*}=R$.

2) The dual $\mathcal{P}$-linear coalgebra of a $\mathcal{P}$-algebra

Fix a $\mathcal{P}$-algebra $R$. We construct a cocommutative $\mathcal{P}$-linear coalgebra $C$ together with a $\mathcal{P}$-linear coalgebra/P-algebra pairing $C \otimes R \longrightarrow \mathcal{P}$. This is done by induction of the degree. Since a right $\mathcal{P}$-action increases the degree, it is necessary to define $S_{m}$-modules $C(m)$ in a first step and to equip these $C(m)$ 's with a right $\mathcal{P}$-action in a second step.

Step 1: Let $C(0)=k$, and assume that there are defined $S_{m}$-modules $C(m)$, for $m<n$, together with a coassociative and cocommutative coproduct

$$
\Delta: C(m) \longrightarrow(C \otimes C)(m), \text { for } m<n,
$$

and a pairing

$$
\langle-,-\rangle: C(m) \otimes R \longrightarrow \mathcal{P}(m), \text { for } m<n,
$$

which makes the coproduct above and the product of the $\mathcal{P}$-algebra adjoint to each other. These conditions make sense, because the degree $m$ component of the tensor product $C \otimes C$ involves only the components of lower degree of $C$.

Whenever defined, $D(m)$ denotes the $S_{m}$-module

$$
D(m):=\bigoplus_{0<p<n} \operatorname{Ind}_{S_{p} \times S_{n-p}}^{S_{n}} C(p) \otimes C(n-p) .
$$

Hence, at this stage, $D(m)$ is defined for all $m \leq n$, and we have

$$
(C \otimes C)(m)=C(m) \otimes 1 \oplus D(m) \oplus 1 \otimes C(m), \text { for all } m<n .
$$

We define $C(n)$ as a sub- $S_{n}$-module of $\operatorname{Mod}_{k}(R, \mathcal{P}(n)) \oplus D(n)$. 
Let us introduce the sequence of $S_{m}$-modules $C^{\prime}(m), m \leq n$, defined by

$$
C^{\prime}(m):= \begin{cases}C(m), & \text { if } m<n, \\ \operatorname{Mod}_{k}(R, \mathcal{P}(n)) \oplus D(n), & \text { if } m=n .\end{cases}
$$

We extend the coproduct of $(C(m), m<n)$ to $\left(C^{\prime}(m), m \leq n\right)$. Let $c \in C^{\prime}(n)$. Hence, we have $c=f \oplus\left(\sum_{i} \sigma^{i} \cdot x^{i} \otimes y^{i}\right)$, where $f \in \operatorname{Mod}_{k}(R, \mathcal{P}(n))$ and $\sum_{i} \sigma^{i}$. $x^{i} \otimes y^{i} \in D(n)$. We have

$$
\left(C^{\prime} \otimes C^{\prime}\right)(n)=C^{\prime}(n) \otimes 1 \oplus D(n) \oplus 1 \otimes C^{\prime}(n)
$$

and we define the coproduct of $c$ as the sum

$$
\Delta(c):=c \otimes 1+\sum_{i} \sigma^{i} \cdot x^{i} \otimes y^{i}+1 \otimes c .
$$

There is also an obvious pairing

$$
\langle-,-\rangle: C^{\prime}(n) \otimes R \longrightarrow \mathcal{P}(n) .
$$

If $c$ is as above and $r \in R$, then we set $\langle c, r\rangle:=f(r)$. We define $C(n)$ as the largest sub- $S_{n}$-module of $C^{\prime}(n)$, on which the coproduct of $C^{\prime}(n)$ is coassociative and cocommutative, and on which the pairing $\langle-,-\rangle: C^{\prime}(n) \otimes R \longrightarrow \mathcal{P}(n)$ makes the coproduct of $C^{\prime}(n)$ adjoint to the product of $R$. Hence, $c$ belongs to $C(n)$, if and only if it satisfies the following identities:

$$
\begin{aligned}
(\Delta \otimes 1 \cdot \Delta)(c) & =(1 \otimes \Delta \cdot \Delta)(c) ; \\
\tau_{*} \Delta(c) & =\Delta(c) ;
\end{aligned}
$$

given a product $\mu\left(r_{1}, \ldots, r_{l}\right) \in R$, in $\mathcal{P}(n)$ we have the identity

$$
\left\langle c, \mu\left(r_{1}, \ldots, r_{l}\right)\right\rangle=\sum_{i} \sigma^{i} \cdot \mu\left(\left\langle c_{(1)}^{i}, r_{1}\right\rangle, \ldots,\left\langle c_{(l)}^{i}, r_{l}\right\rangle\right)
$$

where $\sum_{i} \sigma^{i} \cdot c_{(1)}^{i} \otimes \cdots \otimes c_{(l)}^{i} \in\left(C^{\prime \otimes l}\right)(n)$ denotes an iterated coproduct of $c$. This completes the construction of $C(n)$.

Step 2: To recapitulate, our induction yields an $\mathbf{S}$-module $C$ together with a coassociative and cocommutative coproduct $\Delta: C \longrightarrow C \otimes C$ and a pairing $\langle-,-\rangle: C \otimes R \longrightarrow \mathcal{P}$. Moreover, for $n>0$, we have an embedding

$$
C(n) \hookrightarrow \operatorname{Mod}_{k}(R, \mathcal{P})(n) \oplus D(n) .
$$


We now equip $C$ with a right $\mathcal{P}$-module structure. Assume that $C(m), m<n$, supports a right $\mathcal{P}$-action. More precisely, assume that there are products

$$
C(m) \otimes \mathcal{P}\left(i_{1}\right) \otimes \cdots \otimes \mathcal{P}\left(i_{m}\right) \longrightarrow C\left(i_{1}+\cdots+i_{m}\right)
$$

defined for all integers $i_{1}, \ldots, i_{m}$ and for all $m<n$. Furthermore, we assume that the coproduct $\Delta: C(m) \longrightarrow(C \otimes C)(m)$ and the pairing $\langle-,-\rangle: C(m) \otimes R \longrightarrow$ $\mathcal{P}(m)$ commutes with the right $\mathcal{P}$-action on $C(m)$. Observe that the formula $(2.1)$ for the right $\mathcal{P}$-action on a tensor product equips $(C \otimes C)(m), m<n$, with a canonical right $\mathcal{P}$-action. In fact, the formula $(2.1)$ provides a right $\mathcal{P}$-action on each summand

$$
\operatorname{Ind}_{S_{i} \times S_{m-i}}^{S_{m}} C(i) \otimes C(m-i) \hookrightarrow(C \otimes C)(m)
$$

where $m<n$ and $0 \leq i \leq m$ or $m=n$ and $0<i<n$. In particular, $D(n)=$ $\bigoplus_{0<i<n} \operatorname{Ind}_{S_{i} \times S_{n-i}}^{S_{n}} C(i) \otimes C(n-i)$ supports a right $\mathcal{P}$-action. Our assumptions implies that the right $\mathcal{P}$-action on $\operatorname{Mod}_{k}(R, \mathcal{P})(n) \oplus D(n)$ preserves $C(n)$ (or more exactly, carries an element of $C(n)$ into $\left.C\left(i_{1}+\cdots+i_{n}\right)\right)$.

This achieves the construction: we have a $\mathcal{P}$-linear coalgebra $C$, together with a canonical $\mathcal{P}$-linear coalgebra $/ \mathcal{P}$-algebra pairing

$$
\langle-,-\rangle: C \otimes R \longrightarrow \mathcal{P}
$$

Proof of the adjunction formula: Let $\phi: X \longrightarrow C$ be a morphism of $\mathcal{P}$-linear coalgebras. The associated $\mathcal{P}$-linear coalgebra $/ \mathcal{P}$-algebra pairing $\Phi: X \otimes R \longrightarrow \mathcal{P}$ is defined by $\Phi(x, r):=\langle\phi(x), r\rangle$.

Conversely, let $\Phi: X \otimes R \longrightarrow \mathcal{P}$ be a $\mathcal{P}$-linear coalgebra $/ \mathcal{P}$-algebra pairing. We construct the associated morphism of $\mathcal{P}$-linear coalgebras $\phi: X \longrightarrow C$ by induction on the degree. Assume that there are $S_{m}$-maps $\phi: X(m) \longrightarrow C(m)$, defined for all $m<n$. The pairing $\Phi: X(n) \otimes R \longrightarrow \mathcal{P}(n)$ induces a map $\Phi: X(n) \longrightarrow \operatorname{Mod}(R, \mathcal{P}(n))$. Let $x \in X(n)$. We have

$$
\Delta(x):=x \otimes 1+\sum_{i} \sigma^{i} \cdot x_{(1)}^{i} \otimes x_{(2)}^{i}+1 \otimes x,
$$

where $\sigma^{i} \cdot x_{(1)}^{i} \otimes x_{(2)}^{i} \in \operatorname{Ind}_{S_{p} \times S_{n-p}}^{S_{n}} X(p) \otimes X(n-p)$ for some $p$ such that $0<p<n$. It is easy to see that the element

$$
\Phi(x,-) \oplus\left(\sum_{i} \sigma^{i} \cdot x_{(1)}^{i} \otimes x_{(2)}^{i}\right)
$$

belongs to $C(n) \hookrightarrow \operatorname{Mod}(R, \mathcal{P}(n)) \oplus D(n)$. This achieves the definition of $\phi$ : 
$X \longrightarrow C$. One verifies easily that $\phi$ is a morphism of $\mathcal{P}$-linear coalgebras.

It should be clear that our constructions yield inverse bijections between $\mathcal{B i l}_{\mathcal{P}}(X, R)$ and $\operatorname{coAlg}\left(\operatorname{Mod}_{\mathcal{P}}\right)(X, C)$. Hence, we have the identity

$$
\mathcal{B i l}_{\mathcal{P}}(X, R) \simeq \operatorname{coAlg}\left(\operatorname{Mod}_{\mathcal{P}}\right)(X, C)
$$

and $R^{\circ}=C$.

By adjunction, the dual of a free complete $\mathcal{P}$-algebra is a cofree $\mathcal{P}$-linear coalgebra. More precisely, we have the following result:

2.7. Lemma. Let $V$ be a $k$-module. We have a canonical isomorphism

$$
\operatorname{coAlg}\left(\operatorname{Mod}_{\mathcal{P}}\right)\left(C, C\left(\operatorname{Mod}_{k}(V, \mathcal{P})\right)\right) \simeq \mathcal{B i l}_{\mathcal{P}}(C, \widehat{\mathcal{P}}(V))
$$

and hence, $\widehat{\mathcal{P}}(V)^{\circ}=C\left(\operatorname{Mod}_{k}(V, \mathcal{P})\right)$.

Proof. Recall that we have defined in 2.5 a canonical $\mathcal{P}$-linear coalgebra $/ \mathcal{P}$-algebra pairing

$$
\langle-,-\rangle: C\left(\operatorname{Mod}_{k}(V, \mathcal{P})\right) \otimes \widehat{\mathcal{P}}(V) \longrightarrow \mathcal{P} \text {. }
$$

To any $\mathcal{P}$-linear coalgebra morphism $\phi: C \longrightarrow C\left(\operatorname{Mod}_{k}(V, \mathcal{P})\right)$, we associate the $\mathcal{P}$-linear coalgebra $/ \mathcal{P}$-algebra pairing $\Phi: C \otimes \widehat{\mathcal{P}}(V) \longrightarrow \mathcal{P}$ defined by $\Phi(c, r)=$ $\langle\phi(c), r\rangle$. Conversely, if $\Phi: C \otimes \widehat{\mathcal{P}}(V) \longrightarrow \mathcal{P}$ is a $\mathcal{P}$-linear coalgebra $/ \mathcal{P}$-algebra pairing, then it provides by restriction a $\mathcal{P}$-linear map $\pi: C \longrightarrow \operatorname{Mod}_{k}(V, \mathcal{P})$. Hence, by the universal property of the cofree $\mathcal{P}$-linear coalgebra, we obtain a $\mathcal{P}$-linear coalgebra morphism $\phi: C \longrightarrow C\left(\operatorname{Mod}_{k}(V, \mathcal{P})\right)$. These two constructions are obviously inverse to each other.

2.8. Corollary. Let $\phi: \widehat{\mathcal{P}}(V) \longrightarrow \widehat{\mathcal{P}}(W)$ be a $\mathcal{P}$-algebra morphism. The adjoint morphism $\phi^{\circ}: C\left(\operatorname{Mod}_{k}(W, \mathcal{P})\right) \longrightarrow C\left(\operatorname{Mod}_{k}(V, \mathcal{P})\right)$, is characterized by

$$
\left\langle\phi^{\circ}(c), v\right\rangle=\langle c, \phi(v)\rangle, \forall c \in C\left(\operatorname{Mod}_{k}(W, \mathcal{P})\right), \forall v \in V .
$$

(We consider the pairings defined in 2.5.)

To achieve this section, let us identify the dual $\mathcal{P}$-linear coalgebra of a cogroup. The next statement is an immediate consequence of the adjunction formula. 
2.9. Proposition. We have the identities

$$
\left(\operatorname{colim} R_{i}\right)^{\circ}=\lim R_{i}^{\circ}, \text { and }\left(\operatorname{colim} C_{i}\right)^{*}=\lim C_{i}^{*} .
$$

As an instance of the first identity, the dual $\mathcal{P}$-linear coalgebra functor $(-)^{\circ}$ converts a coproduct in the category of $\mathcal{P}$-algebras into a product in the category of $\mathcal{P}$-linear coalgebras. Furthermore, $(-)^{\circ}$ converts a cogroup in the category of $\mathcal{P}$-algebras into a group in the category of $\mathcal{P}$-linear coalgebras.

But, as in any symmetric monoidal category, the product in the category of cocommutative $\mathcal{P}$-linear coalgebras is given by the right $\mathcal{P}$-module tensor product. Furthermore, the notion of a group in the category of $\mathcal{P}$-linear coalgebras is equivalent to the notion of a cocommutative Hopf algebra in the monoidal category of right $\mathcal{P}$-modules (cf. A.5). Let us recall the definition of this notion:

\subsection{On cocommutative $\mathcal{P}$-linear Hopf algebras}

We call (cocommutative) $\mathcal{P}$-linear Hopf algebra a (cocommutative) Hopf algebra in the monoidal category of right $\mathcal{P}$-modules. Explicitly, a $\mathcal{P}$-linear Hopf algebra $H$ is a $\mathcal{P}$-linear coalgebra equipped with a unital and associative product $m$ : $H \otimes H \longrightarrow H$, which is a morphism of $\mathcal{P}$-linear coalgebras.

Finally, we have obtained

2.11. Proposition. (1) Let $R, S$ be complete $\mathcal{P}$-algebras. We have the identity of $\mathcal{P}$-linear coalgebras

$$
(R \vee S)^{\circ}=R^{\circ} \otimes S^{\circ}
$$

(2) If $R$ is a cogroup object in the category of complete $\mathcal{P}$-algebras, then $R^{\circ}$ is a $\mathcal{P}$-linear Hopf algebra.

\section{$\S 3$. Proof of theorem 0.1}

Throughout this section, we work over a field of characteristic 0 . Let $Q R$ be the $k$-module of indecomposable elements of the $\mathcal{P}$-algebra $R$, defined as the quotient of $R$ by the image of $\oplus_{n \geq 2} \mathcal{P}(n) \otimes_{S_{n}} R^{\otimes n}$ under the product. Choose a section of the canonical projection $R \longrightarrow Q R$. Let us denote by $V$ the image of this section. Since $R$ is complete, by a step by step approximation process, one can show that the $k$-module $V$ generates the $\mathcal{P}$-algebra $R$. Hence, we have $R=\widehat{\mathcal{P}}(V) / I$ for some ideal $I$. We prove that $I$ is zero, and theorem 0.1 follows. 
Since $(-)^{\circ}$ converts colimits into limits, we obtain the equalizer

$$
R^{\circ} \stackrel{i}{\longrightarrow} C\left(\operatorname{Mod}_{k}(V, \mathcal{P})\right) \underset{0}{\stackrel{\pi}{\longrightarrow}} C\left(\operatorname{Mod}_{k}(I, \mathcal{P})\right) .
$$

3.1. Lemma. If $i$ is an isomorphism, then $I=0$.

Proof. If $i$ is an isomorphism, then $\pi=0$. Let $r \in I$. Since $\pi$ is dual to the $\mathcal{P}$-algebra morphism induced by the inclusion $I \hookrightarrow \widehat{\mathcal{P}}(V)$, by corollary 2.8 , we have

$$
\langle c, r\rangle=\langle\pi(c), r\rangle=0, \forall c \in C\left(\operatorname{Mod}_{k}(V, \mathcal{P})\right) .
$$

Let $x_{i}, i \in \mathcal{I}$ be a basis of $V$. We can expand $r$ as a sum of homogeneous monomials $M_{\alpha}$ of multidegree $\alpha$. More precisely, $\alpha$ is a function from the index set to the integers, which is zero except on a finite number of indices, say $1, \ldots, n \in \mathcal{I}$. Let us denote by $\alpha_{i}$ the image of $i$ under $\alpha$ and $N=\alpha_{1}+\cdots+\alpha_{n}$. We have $M_{\alpha_{1}, \ldots, \alpha_{n}}=\mu_{\alpha}\left(x_{1}^{\otimes \alpha_{1}}, \ldots, x_{n}^{\otimes \alpha_{n}}\right)$, for some $\mu_{\alpha} \in \mathcal{P}(N)$. Let $X_{i} \in \operatorname{Mod}_{k}(V, \mathcal{P}(1))$ be the dual basis of the $x_{j}^{\prime} s$ (recall that $\mathcal{P}(1)=k$ ). Let us consider the element

$$
c=\sum_{\sigma \in S_{N}} \frac{1}{\alpha_{1} ! \cdots \alpha_{r} !} \sigma_{*}\left(X_{1}^{\otimes \alpha_{1}}, \ldots, X_{n}^{\otimes \alpha_{n}}\right),
$$

where $\sigma_{*}$ denotes the action of $\sigma \in S_{N}$ on $V^{\otimes N}$ (cf. 1.1.4). This element is obviously $S_{N}$ invariant and hence, belongs to $C\left(\operatorname{Mod}_{k}(V, \mathcal{P})\right)$. By definition, we have

$$
\langle c, r\rangle=\left\langle c, M_{\alpha}\right\rangle=\mu_{\alpha}
$$

Hence, $\langle c, r\rangle=0$ implies $\mu_{\alpha}=0, \forall \alpha$.

In fact, this lemma is the only point where the characteristic assumption is required.

We now need a more tractable description of the equalizer (3.1), as provided by the next lemma.

3.2. Lemma. Let $\pi: C \longrightarrow D$ be a morphism of $\mathcal{P}$-linear coalgebras. Consider the $\mathbf{S}$-submodule $K$ of $C$ defined inductively by

$$
\begin{aligned}
& K(n)= \\
& \left\{c \in C(n), \text { such that } \pi(c)=0 \text { and } \Delta c-c \otimes 1-1 \otimes c \in \oplus \operatorname{Ind}_{S_{i} \times S_{j}}^{S_{n}} K(i) \otimes K(j)\right\}
\end{aligned}
$$


Then $K$ is a sub-P-linear coalgebra of $C$. Moreover, the following diagram is an equalizer

$$
K \stackrel{i}{\longrightarrow} C \underset{0}{\stackrel{\pi}{\longrightarrow}} D
$$

Proof. Let $\phi: X \longrightarrow C$ be a $\mathcal{P}$-linear coalgebra morphism such that $\pi \phi=0$. By an immediate induction process one shows that the image of $\phi$ lies in $K$. This proves the lemma.

3.3. Lemma. The linear component of $C\left(\operatorname{Mod}_{k}(V, \mathcal{P})\right)$ is contained in $R^{\circ}$. Explicitly, we have $\operatorname{Mod}_{k}(V, \mathcal{P}) \hookrightarrow R^{\circ}$.

Proof. Let $f \in \operatorname{Mod}_{k}(V, \mathcal{P})$. Since $\Delta f=f \otimes 1+1 \otimes f$, we only need to prove that $\pi(f)=0$. Since $V$ consists in indecomposable elements, $I$ is contained in $\prod_{n \geq 2} \mathcal{P}(n) \otimes_{S_{n}} V^{\otimes n}$. Thus, the lemma is an immediate consequence of corollary 2.8 .

3.4. Proof of theorem 0.1. If $R$ is a cogroup object, then $R^{\circ}$ is a $\mathcal{P}$-linear Hopf algebra. Let $X$ be the primitive part of $R^{\circ}$. By the general theory of Hopf algebras in a monoidal category, the embedding $X \hookrightarrow R^{\circ}$ extends to an isomorphism of coalgebras from the cofree coalgebra $C(X)$ to $R^{\circ}$ (cf. theorem A.9).

By the previous lemma, $\operatorname{Mod}_{k}(V, \mathcal{P}) \hookrightarrow R^{\circ}$. Moreover, any element of $\operatorname{Mod}_{k}(V, \mathcal{P})$ is clearly primitive. Hence, we have $\operatorname{Mod}_{k}(V, \mathcal{P}) \hookrightarrow X$, and this map induces an embedding of $\mathcal{P}$-linear coalgebras $C\left(\operatorname{Mod}_{k}(V, \mathcal{P})\right) \hookrightarrow C(X)=R^{\circ}$.

Furthermore, the composite

$$
C\left(\operatorname{Mod}_{k}(V, \mathcal{P})\right) \hookrightarrow R^{\circ} \stackrel{i}{\longrightarrow} C\left(\operatorname{Mod}_{k}(V, \mathcal{P})\right) \longrightarrow \operatorname{Mod}_{k}(V, \mathcal{P})
$$

is the canonical projection of $C\left(\operatorname{Mod}_{k}(V, \mathcal{P})\right)$ onto $\operatorname{Mod}_{k}(V, \mathcal{P})$. It follows that $i: R^{\circ} \longrightarrow C\left(\operatorname{Mod}_{k}(V, \mathcal{P})\right)$ has a section. Hence, $i$ is necessarily an isomorphism, and by lemma 3.1, this completes the proof of the theorem.

\section{$\S 4$. Groups in coalgebras over an operad are cofree coalge- bras}

In this section, we give a sketch of the proof of theorem 0.3. As mentioned in the introduction, we deduce theorem 0.3 from a variant of theorem 0.1 . For simplicity, we work with ungraded objects. As explained earlier, it is easy to find the graded analogues of our statements. 
Throughout this section, we work over a fixed unital operad $\mathcal{P}$, with all $k$ modules $\mathcal{P}(n)$ finite dimensional.

4.1. Coalgebras over an operad (cf. [23])

A $\mathcal{P}$-coalgebra $C$ is a $k$-module together with $S_{n}$-equivariant morphisms $\rho_{n}: \mathcal{P}(n) \otimes$ $C \longrightarrow C^{\otimes n}$, which are associative with respect to the operad product. More precisely, these morphisms make the following diagram commute

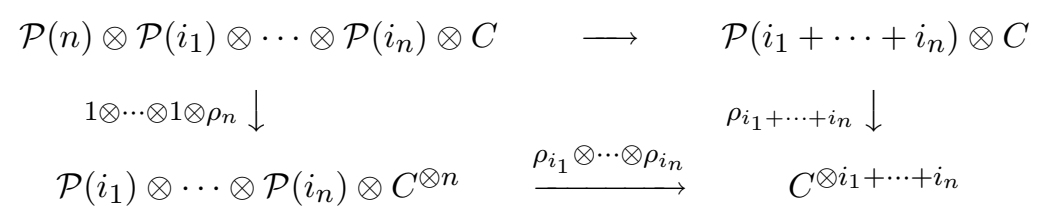

Let $\mu \in \mathcal{P}(n), c \in C$. We denote by $\mu^{*}(c)$ the image of $\mu \otimes c$ under the $\mathcal{P}$-coalgebra coproduct $\rho_{n}$.

\subsection{Connected $\mathcal{P}$-coalgebras}

A $\mathcal{P}$-coalgebra $C$ is called connected, if its coproduct verifies the property

$$
\forall c \in C, \exists N \text { such that } \forall \mu \in \mathcal{P}(n), n>N \Longrightarrow \mu^{*}(c)=0 .
$$

4.3. Remark. Recall that a graded object $V$ is called connected, when $V_{d}=0$ for $d \leq 0$. Let $C$ be a graded $\mathcal{P}$-coalgebra. If $C$ is connected (as a graded object), then any element of degree $d$ is necessarily cancelled by the coproduct $\mathcal{P}(n) \otimes C \longrightarrow C^{\otimes n}$, as soon as $n>d$. Hence, $C$ is connected in the sense defined above.

\subsection{Cooperads and connected $\mathcal{P}$-coalgebras}

Let $\mathcal{P}^{*}$ denote the $k$-linear dual of $\mathcal{P}$, equipped with the adjoint $\mathbf{S}$-module structure. As each $k$-module $\mathcal{P}(n)$ is finite dimensional, $\mathcal{P}^{*}$ is equipped with coproducts $\mathcal{P}^{*}\left(i_{1}+\cdots+i_{n}\right) \longrightarrow \mathcal{P}^{*}(n) \otimes \mathcal{P}^{*}\left(i_{1}\right) \otimes \cdots \otimes \mathcal{P}^{*}\left(i_{n}\right)$, which make it into a cooperad (the dual notion of an operad). As for operads, these coproducts make the functor

$$
C(\mathcal{P}, V):=\bigoplus_{n=0}^{\infty}\left(\mathcal{P}^{*}(n) \otimes V^{\otimes n}\right)^{S_{n}}
$$

into a comonad on $k$-modules (cf. [14]). In fact, a connected $\mathcal{P}$-coalgebra is equivalent to a coalgebra over this comonad. In particular, if $V$ denotes a $k$ module, then $C(\mathcal{P}, V)$ is the cofree $\mathcal{P}$-coalgebra cogenerated by $V$. 


\subsection{Groups in coalgebras over an operad}

The category of $\mathcal{P}$-coalgebras is equipped with a product, denoted by $C_{1} \times C_{2}$, whose underlying $k$-module is given by the equalizer

$$
C_{1} \times C_{2} \longrightarrow C\left(\mathcal{P}, C_{1} \oplus C_{2}\right) \underset{\delta_{1}}{\stackrel{\delta_{0}}{\longrightarrow}} C\left(\mathcal{P}, C\left(\mathcal{P}, C_{1}\right) \oplus C\left(\mathcal{P}, C_{2}\right)\right)
$$

Let $\rho_{k}: C_{k} \longrightarrow C\left(\mathcal{P}, C_{k}\right)$ be induced by the coproduct of the $\mathcal{P}$-coalgebra $C_{k}$, $k=1,2$. As for the coproduct of algebras over an operad, the map $\delta_{0}$ is defined by $\delta_{0}=C\left(\mathcal{P}, \rho_{1} \oplus \rho_{2}\right)$, and $\delta_{1}$ is the composite of the comonad coproduct with the canonical projection:

$$
C\left(\mathcal{P}, C_{1} \oplus C_{2}\right) \longrightarrow C\left(\mathcal{P}, C\left(\mathcal{P}, C_{1} \oplus C_{2}\right)\right) \longrightarrow C\left(\mathcal{P}, C\left(\mathcal{P}, C_{1}\right) \oplus C\left(\mathcal{P}, C_{2}\right)\right)
$$

A group in the category of connected $\mathcal{P}$-coalgebras is a $\mathcal{P}$-coalgebra $C$ equipped with a "product" $\gamma: C \times C \longrightarrow C$ and an "antipode" $\iota: C \longrightarrow C$ satisfying the classical identities.

We introduce the notion of a pseudocompact $\mathcal{P}$-algebra. Then, we show that the category of $\mathcal{P}$-coalgebras is dual to the category of pseudocompact $\mathcal{P}$-algebras.

4.6. Definition. Let $V$ be a $k$-module. A topology on $V$ is given by a poset of submodules $\left(V_{i}, i \in \mathcal{I}\right)$. We assume that each $k$-module $V_{i}$ has a finite codimension. Moreover, the indexing poset $\mathcal{I}$ is supposed to be filtering. Explicitly, $\mathcal{I}$ satisfies the assumption

$$
\forall i, j \in \mathcal{I}, \exists k \in \mathcal{I} \text { such that } i, j \leq k
$$

The topological module $V$ is called separated if $\cap_{i} V_{i}=0$, and pseudocompact if in addition $V=\lim V / V_{i}$.

Of course, any separated topological $k$-module $\left(V ; V_{i}, i \in \mathcal{I}\right)$ can be completed into a pseudocompact $k$-module $\left(\widehat{V} ; \widehat{V}_{i}, i \in \mathcal{I}\right)$. In fact, we set

$$
\begin{aligned}
\widehat{V} & :=\lim V / V_{i} \\
\widehat{V}_{i} & :=\lim _{i \leq j} V_{i} / V_{j}
\end{aligned}
$$

By the Mittag-Leffler theorem (see [7]), we have $\widehat{V} / \widehat{V}_{i}=V / V_{i}$. Hence $\widehat{V}_{i}$ has a finite codimension and $\left(\widehat{V} ; \widehat{V}_{i}, i \in \mathcal{I}\right)$ is complete.

Let $\left(V_{i}, i \in \mathcal{I}\right),\left(W_{j}, j \in \mathcal{J}\right)$ be topological $k$-modules. A linear map $u: V \longrightarrow$ $W$ is continuous, if it satisfies the following property

$$
\forall j \in \mathcal{J}, \exists i \in \mathcal{I} \text { such that } u\left(V_{i}\right) \subset W_{j}
$$


Let $V$ be a topological $k$-module. We denote by $V^{\prime}$ the topological dual of $V$. That is, the $k$-module of linear forms which vanish over a certain $V_{i}$ (also known to be the continuous linear forms). Let $E$ be a $k$-module. We equip the $k$-linear dual module $E^{*}$ with the topology given by the poset of the $k$-modules $V^{\perp}$, where $V$ ranges over the set of finite dimensional submodules of $E$.

The following proposition is well known:

4.7. Proposition. The functors $(-)^{*}$ and $(-)^{\prime}$ are inverse to each other and induce an equivalence of categories between the category of $k$-modules and the category of pseudocompact $k$-modules together with continuous linear maps.

Moreover, if $V$ is a separated topological $k$-module, then $V^{\prime *}$ is isomorphic to the completion of $V$.

4.8. Theorem. Let $p: V \longrightarrow W$ be a surjective continuous linear map. Assume that $V$ is pseudocompact and that $W$ is separated. Let $i: U \longrightarrow V$ be the kernel of $p$. We equip the $k$-module $U$ with the induced topology. We claim that:

(1) the $k$-modules $U$ and $W$ are pseudocompact,

(2) the map $p$ has a continuous section.

Proof. Let $\left(V_{i}, i \in \mathcal{I}\right)\left(\right.$ resp. $\left.\left(W_{j}, j \in \mathcal{J}\right)\right)$ define the topology of $V$ (resp. $W$ ).

Let us prove that $U$ is pseudocompact. In fact, we show that $U$ is closed in $V$. This statement is equivalent to the identity $\cap_{i}\left(V_{i}+U\right)=U$. Let $u \in \cap_{i}\left(V_{i}+U\right)$. Given $j \in \mathcal{J}$, there exists $i$ such that $p\left(V_{i}\right) \subset W_{j}$, because $p$ is continuous. As a consequence, $p\left(V_{i}+U\right) \subset W_{j}$ and $p(u) \in W_{j}$. Hence, $p(u)$ belongs to the intersection $\cap_{j} W_{j}$, which is zero since $W$ is assumed to be separated.

The map $i^{\prime}$ is surjective, since the functor $(-)^{\prime}$ is an equivalence of categories. Let $k: K \longrightarrow V^{\prime}$ be the kernel of $i^{\prime}$. Pick a splitting in the exact sequence $0 \longrightarrow K \longrightarrow V^{\prime} \longrightarrow U^{\prime} \longrightarrow 0$. Since $V^{\prime *}=V$ and $U^{\prime *}=U$, we obtain a split exact sequence

$$
U \underset{i}{\stackrel{r}{\leftrightarrows}} V \underset{q}{\stackrel{s}{\leftrightarrows}} K^{*} .
$$

The map $\phi=p s$ is a continuous bijection from $K^{*}$ into $W$. Let us prove that $\phi$ is open, and hence, is an isomorphism between $W$ and $K^{*}$. (This assertion immediately implies that $W$ is pseudocompact and has a section in $V$.)

Fix $j \in \mathcal{J}$. We show that there exists a finite dimensional subspace of $K$, denoted by $E_{j}$, such that $E_{j}^{\perp}=\phi^{-1} W_{j}$. Since $\phi$ is continuous, there exists $F \hookrightarrow K$ finite dimensional and such that $F^{\perp} \subset \phi^{-1} W_{j}$. Let

$$
E:=\left\{x \in K, \text { such that }\langle k, x\rangle=0, \forall k \in \phi^{-1} W_{j}\right\} .
$$

We have $F^{\perp} \subseteq \phi^{-1} W_{j} \subset E^{\perp}$. Hence, $E \subseteq F^{\perp \perp}=F$ and $E$ is finite dimensional. 
Clearly, $E$ is the orthogonal complement of $\phi^{-1} W_{j} / F^{\perp}$ in $F$. Hence, we have $\phi^{-1} W_{j} / F^{\perp}=E^{\perp} / F^{\perp}$, and it follows $\phi^{-1} W_{j}=E^{\perp}$.

Since $W$ is separated, we have $\cap_{j} W_{j}=0$ and hence $\sum_{j} E_{j}=K$. Let $E$ be a finite dimensional subspace of $K$. Necessarily, $E \subset E_{j_{1}}+\cdots+E_{j_{n}}$ for some indices $j_{1}, \ldots, j_{n}$. As $\mathcal{J}$ is filtering, there exists $j \in \mathcal{J}$ larger than $j_{1}, \ldots, j_{n}$. Then, we have

$$
\phi^{-1} W_{j} \subset \phi^{-1} W_{j_{1}} \cap \cdots \cap \phi^{-1} W_{j_{n}}=\left(E_{j_{1}}+\cdots+E_{j_{n}}\right)^{\perp} \subset E^{\perp} .
$$

This proves that $\phi$ is open.

\subsection{The completed tensor product}

Let $\left(V ; V_{i}, i \in \mathcal{I}\right),\left(W ; W_{j}, j \in \mathcal{J}\right)$ be topological $k$-modules. The product set $\mathcal{I} \times \mathcal{J}$ is equipped with the order defined by $(i, j) \leq(k, l)$ if $i \leq k$ and $j \leq l$. We equip the tensor product $V \otimes W$ with the topology given by

$$
(V \otimes W)_{i, j}=V_{i} \otimes W+V \otimes W_{j},(i, j) \in \mathcal{I} \times \mathcal{J} .
$$

The completion of $V \otimes W$ with respect to this topology is known as the completed tensor product and is denoted by $V \widehat{\otimes} W$.

Notice that the completed tensor product behaves well with respect to the duality functors, as shown by the next lemma.

4.10. Lemma. Let $V, W$ be topological k-modules. We have $V^{\prime} \otimes W^{\prime} \simeq(V \widehat{\otimes} W)^{\prime}$. Let $E, F$ be $k$-modules. We have $E^{*} \widehat{\otimes} F^{*} \simeq(E \otimes F)^{*}$.

Proof. Elementary linear algebra.

\subsection{Pseudocompact $\mathcal{P}$-algebras}

A pseudocompact $\mathcal{P}$-algebra is a pseudocompact $k$-module $R$ together with a compatible $\mathcal{P}$-algebra structure. More precisely, the $\mathcal{P}$-algebra products

$$
\mathcal{P}(n) \otimes R^{\otimes n} \longrightarrow R
$$

are assumed to be continuous with respect to the topology on the tensor product introduced above. We make the following additional assumption. Recall that $R^{(N)}$ is the $N$-th power of the augmentation ideal of $R$ (see definition 1.1.8). Let $R_{i}, i \in \mathcal{I}$ denote the topology of $R$. Fix $i \in \mathcal{I}$. We assume that $R^{(N)}$ is contained in $R_{i}$ for $N$ large. 
4.12. Example. Let $V$ be a pseudocompact $k$-module. Equip the $\mathcal{P}$-algebra $T(\mathcal{P}, V)$ with the topology given by the poset of ideals

$$
I_{(i, N)}=\left(\bigoplus_{n=1}^{N} \mathcal{P}(n) \otimes_{S_{n}}\left(\sum V \otimes \cdots \otimes V_{i} \otimes \cdots \otimes V\right)\right) \oplus\left(\bigoplus_{n>N} \mathcal{P}(n) \otimes_{S_{n}} V^{\otimes n}\right) .
$$

Completing $T(\mathcal{P}, V)$ with respect to this poset of ideals we obtain a pseudocompact $\mathcal{P}$-algebra, which we denote by $\widehat{\mathcal{P}}_{c}(V)$. We claim that $\widehat{\mathcal{P}}_{c}(V)$ is the free pseudocompact $\mathcal{P}$-algebra generated by $V$. Let $\phi: V \longrightarrow R$ be a continuous morphism. In fact, it suffices to show that the induced $\mathcal{P}$-algebra morphism $\phi: T(\mathcal{P}, V) \longrightarrow R$ is continuous. Assume that the topology on $R$ is given by $\left(R_{j}, j \in \mathcal{J}\right)$. Fix $j \in \mathcal{J}$. By hypothesis, there exists $N$ such that $R^{(N+1)} \subset R_{j}$. Hence, $\oplus_{n>N} \mathcal{P}(n) \otimes_{S_{n}} V^{\otimes n}$ is mapped into $R_{j}$. By continuity, we can pick $j^{\prime} \in \mathcal{J}$ such that the $\mathcal{P}$-algebra product maps $\mathcal{P}(n) \otimes R \otimes \cdots \otimes R_{j^{\prime}} \otimes \cdots \otimes R$ into $R_{j}$. Now pick $i$ such that $\phi\left(V_{i}\right) \subset R_{j^{\prime}}$. The ideal $I_{(i, N)}$ is mapped into $R_{j}$. Thus, we are done.

Notice that we have

$$
\widehat{\mathcal{P}}_{c}(V)=\prod_{n=0}^{\infty} \mathcal{P}(n) \otimes_{S_{n}} V^{\widehat{\otimes} n} .
$$

In the same way, it is straightforward to check that the coproduct of two pseudocompact $\mathcal{P}$-algebras is given by the usual coequalizer

$$
\widehat{\mathcal{P}}_{c}\left(\widehat{\mathcal{P}}_{c}(R) \oplus \widehat{\mathcal{P}}_{c}(S)\right) \Longrightarrow \widehat{\mathcal{P}}_{c}(R \oplus S) \longrightarrow R \vee S .
$$

We have also a notion of a cogroup in the pseudocompact $\mathcal{P}$-algebras.

\subsection{On $\mathcal{P}$-coalgebra/ $\mathcal{P}$-algebra pairings}

A pairing between a connected $\mathcal{P}$-coalgebra $C$ and a pseudocompact $\mathcal{P}$-algebra $R$ is a $k$-linear map

$$
\langle-,-\rangle: C \otimes R \longrightarrow k,
$$

which makes the coproduct of $C$ adjoint to the product of $R$. Explicitly, we have the relation

$$
\left\langle\mu\left(f_{1}, \ldots, f_{n}\right), c\right\rangle=\left\langle f_{1} \otimes \cdots \otimes f_{n}, \mu^{*}(c)\right\rangle, \forall \mu \in \mathcal{P}(n), \forall f_{1}, \ldots, f_{n} \in R, \forall c \in C .
$$

The pairing $\langle-,-\rangle$ is continuous if it satisfies the property

$$
\forall c \in C, \exists i \text { such that } \forall r \in R_{i},\langle c, r\rangle=0 .
$$


We denote by $\mathcal{B} i l_{k}^{\text {cont }}(C, R)$ the $k$-module of continuous $\mathcal{P}$-coalgebra $/ \mathcal{P}$-algebra pairings between $C$ and $R$.

4.14. Theorem. Let $\widehat{\mathcal{P}}_{c}-A l g$ denote the category of pseudocompact $\mathcal{P}$-algebras. Let $\mathcal{P}-$ coAlg denote the category of connected $\mathcal{P}$-coalgebras. There exists a pair of contravariant adjoint functors

$$
(-)^{\prime}: \widehat{\mathcal{P}}_{c}-A l g \rightleftarrows \mathcal{P}-\operatorname{coAlg}:(-)^{*},
$$

which both represent the bifunctor $\mathcal{B i l}_{k}^{\mathrm{cont}}(C, R)$. More precisely, we have canonical isomorphisms

$$
\widehat{\mathcal{P}}_{c}-\operatorname{Alg}\left(R, C^{*}\right) \simeq \mathcal{B}_{i}^{\text {cont }}(C, R) \simeq \mathcal{P}-\operatorname{coAlg}\left(C, R^{\prime}\right) .
$$

Moreover, these functors $(-)^{\prime}$ and $(-)^{*}$ are inverse to each other.

Proof. We give only the construction of the duality functors. The rest of the proof reduces to straightforward verifications. Let $C$ be a connected $\mathcal{P}$-coalgebra. The $k$-linear dual module $C^{*}$ is equipped with a pseudocompact $\mathcal{P}$-algebra structure. The product $\mathcal{P}(n) \otimes C^{* \otimes n} \longrightarrow C^{*}$ is defined by

$$
\left\langle\mu\left(f_{1}, \ldots, f_{n}\right), c\right\rangle:=\left\langle f_{1} \otimes \cdots \otimes f_{n}, \mu^{*}(c)\right\rangle, \mu \in \mathcal{P}(n), f_{1}, \ldots, f_{n} \in C^{*}, c \in C .
$$

Let $V$ be a finite dimensional submodule of $C$. The image of $V$ under the coproduct $\mathcal{P}(n) \otimes C \longrightarrow C^{\otimes n}$ is necessarily finite dimensional, hence is contained in $V_{1} \otimes$ $\cdots \otimes V_{n}$ for some finite dimensional submodules $V_{1}, \ldots, V_{n}$. The product defined above maps $\sum \mathcal{P}(n) \otimes C^{*} \otimes \cdots \otimes V_{i}^{\perp} \otimes \cdots \otimes C^{*}$ into $V^{\perp}$, and hence is continuous. Moreover, as $V$ is finite dimensional, $V$ is cancelled by $C^{* \otimes N}$ for $N$ large (recall that $C$ is assumed to be connected). This completes the definition of the functor $(-)^{*}: \mathcal{P}-\operatorname{coAlg} \longrightarrow \widehat{\mathcal{P}}_{c}-$ Alg.

As a consequence of lemma 4.10 , if $R$ is a pseudocompact $\mathcal{P}$-algebra, then its topological $k$-linear dual is equipped with a $\mathcal{P}$-coalgebra structure. The $\mathcal{P}$ coalgebra coproduct is defined by

$$
R^{\prime} \longrightarrow\left(\mathcal{P}(n) \otimes R^{\widehat{\otimes} n}\right)^{\prime} \simeq \mathcal{P}(n)^{*} \otimes R^{\prime \otimes n} .
$$

As a consequence of theorem 4.14, a $\mathcal{P}$-coalgebra is a group object if and only if its dual $\mathcal{P}$-algebra is a cogroup object. Moreover, a $\mathcal{P}$-coalgebra is cofree if and only if its dual $\mathcal{P}$-algebra is free. Thus, as claimed in the introduction, theorem 0.3 is a consequence of the following statement which is the pseudocompact variant of theorem 0.1 . 
4.15. Theorem. Assume the ground field $k$ to be of characteristic zero. Let $\mathcal{P}$ be a unital operad. Assume that each $k$-module $\mathcal{P}(n)$ is finite dimensional. If $R$ is a pseudocompact $\mathcal{P}$-algebra equipped with a cogroup structure, then $R$ is a free pseudocompact $\mathcal{P}$-algebra.

We show only how we adapt the methods involved in section 2 and section 3 in order to work with pseudocompact $\mathcal{P}$-algebras. The plan of the proof remains the same. In general, we have just to make some additional continuity assumptions.

For instance, let $V$ be a pseudocompact $k$-module. We denote by $\operatorname{Mod}_{k}^{\text {cont }}(V, \mathcal{P})$ the $k$-module of continuous linear maps from $V$ to $\mathcal{P}$, (the maps which vanish over a certain $V_{i}$ ). When we deal with pseudocompact $\mathcal{P}$-algebras, we replace $\operatorname{Mod}_{k}(V, \mathcal{P})$ by $\operatorname{Mod}_{k}^{\text {cont }}(V, \mathcal{P})$ in the definitions of section 2 and section 3.

In the same way, we have to consider continuous $\mathcal{P}$-linear coalgebra $/ \mathcal{P}$-algebra pairings between a $\mathcal{P}$-linear coalgebra $C$ and a pseudocompact $\mathcal{P}$-algebra $R$. Such a pairing is a $\mathcal{P}$-linear map $\langle-,-\rangle: C \otimes R \longrightarrow \mathcal{P}$ which makes the coproduct of $C$ adjoint to the product of $R$ and which satisfies the following continuity property

$$
\forall c \in C, \exists i \in \mathcal{I} \text {, such that } r \in R_{i} \Longrightarrow\langle c, r\rangle=0 .
$$

We denote by $\mathcal{B} i l_{\mathcal{P}}^{\text {cont }}(C, R)$ the $k$-module of continuous $\mathcal{P}$-linear coalgebra $/ \mathcal{P}$ algebra pairings between $C$ and $R$. As in 2.5, there is a canonical continuous $\mathcal{P}$-linear coalgebra $/ \mathcal{P}$-algebra pairing

$$
\langle-,-\rangle: C\left(\operatorname{Mod}_{k}^{\text {cont }}(V, \mathcal{P})\right) \otimes \widehat{\mathcal{P}}_{c}(V) \longrightarrow \mathcal{P} .
$$

In the same way, it is easy to adapt the construction of the dual $\mathcal{P}$-linear coalgebra in the proof of theorem 2.6 to the case of a pseudocompact $\mathcal{P}$-algebra: once we have $S_{m}$-modules $C(m)$, for $m<n$, we define $C(n)$ as a particular sub- $S_{n^{-}}$ module of the sum $\operatorname{Mod}_{k}^{\text {cont }}(R, \mathcal{P}) \oplus D(n)$ where $D(n)=\bigoplus_{0<p<n} \operatorname{Ind}_{S_{p} \times S_{n-p}}^{S_{n}} C(p)$ $\otimes C(n-p)$.

Let $C$ be a $\mathcal{P}$-linear coalgebra. The dual $\mathcal{P}$-algebra of $C$ is equipped the structure of a pseudocompact $\mathcal{P}$-algebra. Recall that $C^{*}$ is $\operatorname{Mod}_{\mathcal{P}}(C, \mathcal{P})$, the $k$ module of $\mathcal{P}$-linear maps from $C$ to $\mathcal{P}$. Let $V$ be a finitely generated sub-right- $\mathcal{P}$ module of $C$. Consider the $k$-module

$$
V^{\perp}:=\left\{f \in \operatorname{Mod}_{\mathcal{P}}(C, \mathcal{P}) \text { such that }\left.f\right|_{V}=0\right\} .
$$

Hence, we have an exact sequence

$$
0 \longrightarrow V^{\perp} \longrightarrow \operatorname{Mod}_{\mathcal{P}}(C, \mathcal{P}) \longrightarrow \operatorname{Mod}_{\mathcal{P}}(V, \mathcal{P}) \longrightarrow 0 .
$$

Since $V$ is finitely generated, the $k$-module $\operatorname{Mod}_{\mathcal{P}}(V, \mathcal{P})$ is finite dimensional and $V^{\perp}$ has a finite codimension. Furthermore, $V^{\perp}$ contains $C^{*(N)}$, when $N$ is large. 
Finally, the poset given by the $V^{\perp}$ 's, where $V$ ranges over the finitely generated sub-right- $\mathcal{P}$-modules of $C$, makes $C^{*}$ into a pseudocompact $\mathcal{P}$-algebra.

Taking these changes into account, theorem 2.6 becomes

4.16. Theorem. The functors defined above

$$
(-)^{\circ}: \widehat{\mathcal{P}}_{c}-\operatorname{Alg} \rightleftarrows \operatorname{coAlg}\left(\operatorname{Mod}_{\mathcal{P}}\right):(-)^{*},
$$

are adjoint to each other and both represent the bifunctor $\mathcal{B} i l_{\mathcal{P}}^{\text {cont }}(C, R)$. More precisely, we have canonical isomorphisms

$$
\widehat{\mathcal{P}}_{c}-\operatorname{Alg}\left(R, C^{*}\right) \simeq \mathcal{B i l}_{\mathcal{P}}^{\text {cont }}(C, R) \simeq \operatorname{coAlg}\left(\operatorname{Mod}_{\mathcal{P}}\right)\left(C, R^{\circ}\right) .
$$

We have also

4.17. Lemma. Let $V$ be a pseudocompact $k$-module. We have a canonical isomorphism

$$
\operatorname{coAlg}\left(\operatorname{Mod}_{\mathcal{P}}\right)\left(C, C\left(\operatorname{Mod}_{k}^{\text {cont }}(V, \mathcal{P})\right)\right) \simeq \mathcal{B}_{l_{\mathcal{P}}}^{\text {cont }}\left(C, \widehat{\mathcal{P}}_{c}(V)\right)
$$

and hence, $\widehat{\mathcal{P}}_{c}(V)^{\circ}=C\left(\operatorname{Mod}_{k}^{\text {cont }}(V, \mathcal{P})\right)$.

The definition of the $k$-module of indecomposables is much more delicate than in the discrete case. As for $T(\mathcal{P}, V)$, the $k$-module

$$
T^{\geq 2}(\mathcal{P}, V)=\bigoplus_{n \geq 2} \mathcal{P}(n) \otimes_{S_{n}} V^{\otimes n}
$$

is equipped with a canonical topology. Actually, after completion, we obtain $\widehat{\mathcal{P}}_{c}^{\geq 2}(V)$, the kernel of the canonical projection $\widehat{\mathcal{P}}_{c}(V) \longrightarrow V$.

Let $R$ be a pseudocompact $\mathcal{P}$-algebra. The $\mathcal{P}$-algebra product induces a canonical continuous morphism $T^{\geq 2}(\mathcal{P}, R) \longrightarrow R$. The closure of the image of this morphism is denoted by Dec $R$ and is known as the $k$-module of (topologically) decomposable elements. The $k$-module of indecomposable elements is defined by $Q R:=R / \operatorname{Dec} R$.

By theorem 4.8, $Q R$ is pseudocompact and the exact sequence

$$
0 \longrightarrow \operatorname{Dec} R \longrightarrow R \longrightarrow Q R \longrightarrow 0
$$

is split. Let $V$ be a continuous section of $Q R$ in $R$. Theorem 4.8 ensures that $V$ 
is pseudocompact.

4.18. Lemma. The continuous map induced by the $\mathcal{P}$-algebra product $\widehat{\mathcal{P}}_{c}(V) \longrightarrow$ $R$ is surjective.

Proof. By theorem 4.8, the image of this map is closed in $R$. Thus, it is sufficient to show that the image of $T(\mathcal{P}, V)$ is dense. Fix $i \in \mathcal{I}$. Let $r \in R$. Since $V$ is a section of $Q R$, there exists $v \in V$ such that $r-v \in \operatorname{Dec} R$. Hence, modulo $R_{i}$, the element $r-v$ is a sum of elements of the form $\mu\left(r_{1}, \ldots, r_{n}\right)$ such that $n \geq 2$. By induction, we obtain an element $s \in T(\mathcal{P}, V)$ such that $r-s$ belongs to $R^{(N)}$ modulo $R_{i}$. Since $R^{(N)} \subseteq R_{i}$ for $N$ large, we are done.

As a consequence, we obtain an exact sequence

$$
0 \longrightarrow I \longrightarrow \widehat{\mathcal{P}}_{c}(V) \longrightarrow R \longrightarrow 0 .
$$

Moreover, the ideal $I$ is contained in $\widehat{\mathcal{P}}_{\bar{c}}^{\geq 2}(V)$. In fact, the commutative diagram

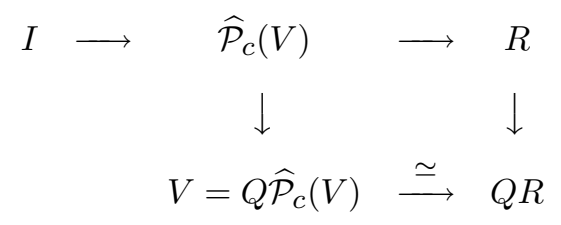

shows that the projection of $I$ onto $V$ is null.

The rest of the proof does not present more difficulties: as in section 3 , we have $R^{\circ}=C\left(\operatorname{Mod}_{k}^{\operatorname{cont}}(V, \mathcal{P})\right)$ and we deduce that $I$ is zero.

\section{$\S 5$. Applications}

In this section we give some examples of cogroups in graded algebras over an operad.

Let $\mathcal{P}=\mathcal{A} s$ be the operad of associative algebras (without unit). In this case, the coproduct is given by

$$
R \vee S=R \oplus S \oplus(R \otimes S) \oplus(S \otimes R) \oplus(R \otimes S \otimes R) \oplus(S \otimes R \otimes S) \oplus \cdots
$$

The free associative algebra is given by the augmentation ideal of the tensor algebra. Cogroups in graded associative algebras are considered by I. Berstein in [1]. 
I. Berstein proved theorem 0.2 in this context.

The Bott-Samelson theorem, which states that

$$
H_{*}(\Omega \Sigma X, k)=T H_{*}(X, k),
$$

where $X$ denotes a connected topological space, occurs as a topological illustration of Berstein theorem.

For the operad $\mathcal{P}=\mathcal{L}$ ie of Lie algebras, the coproduct takes a more complicated form. Nevertheless, as any Lie algebra is the primitive part of its enveloping algebra, the coproduct of two Lie algebras can be written by means of the associative coproduct. More precisely, we have $L_{1} \vee L_{2}=\operatorname{Prim} U\left(L_{1}\right) \vee U\left(L_{2}\right)$, the coproduct of $U\left(L_{1}\right) \vee U\left(L_{2}\right)$ being induced by the coproduct of $U\left(L_{1}\right)$ and the coproduct of $U\left(L_{2}\right)$. In characteristic zero, the free Lie algebra is given by the primitive part of the tensor algebra (cf. [21]). In this case, theorem 0.1 and theorem 0.2 take the following form:

5.1. Theorem. Assume the ground field $k$ to be of characteristic zero. If $L$ is a complete Lie algebra (resp. connected graded Lie algebra) equipped with a cogroup structure, then $L$ is the completion of a free Lie algebra (resp. a free graded Lie algebra).

This result does not seem to occur explicitly in the literature. Notice that this instance of theorem 0.1 could be easily deduced from the associative case.

The case of dual Leibniz algebras was handled by J.M. Oudom [19]. Following Oudom, we explain how theorem 0.3 applies to the computation of the homology of the Lie algebra of matrices.

It is well known that there is an isomorphism relating cyclic homology to the Lie homology of matrices. More precisely we have

$$
H_{*}(g l(A))=\Lambda H C_{*-1}(A)
$$

(cf. [13], [26], [11]). The proof of this theorem is divided in two steps. One calculates the primitive part of $H_{*}(g l(A))$, and then applies the Milnor-Moore theorem. Recently, J.-L. Loday has constructed a non-commutative homology, denoted by $H L_{*}$, such that

$$
H L_{*}(g l(A))=T H H_{*-1}(A)
$$

(cf. [3], [11]). The calculation of the primitive part of $H L_{*}(g l(A))$ was performed by Cuvier. But, since $H L_{*}(g l(A))$ failed to be a bialgebra, there was a lack of general methods for completing the proof, which was achieved in a different manner by J.-L. Loday. 
In [19], J.-M. Oudom proposes a different approach in order to palliate the lack of a Milnor-Moore theorem for $H L_{*}(g l(A))$. It turns out that $H L_{*}(g l(A))$ is equipped with a particular coalgebra structure. Namely, $H L_{*}(g l(A))$ is equipped with the structure of a graded dual Leibniz coalgebra. In fact, J.-M. Oudom proved theorem 0.2 in the particular case of the operad of dual Leibniz algebras. The homology coalgebra $H L_{*}(g l(A))$ is a group in the category of graded dual Leibniz coalgebras. The product of $H L_{*}(g l(A))$

$$
H L_{*}(g l(A)) \times H L_{*}(g l(A)) \longrightarrow H L_{*}(g l(A))
$$

is induced by the direct sum of matrices $g l(A) \oplus g l(A) \longrightarrow g l(A)$. Since the underlying vector space of the free dual Leibniz algebra is given by the tensor algebra, Cuvier's calculation of Prim $H L_{*}(g l(A))$ and theorem 0.3 implies the Cuvier-Loday theorem.

\section{Appendix. Hopf algebras in a symmetric monoidal category}

\section{A.1. Graded $k$-linear symmetric monoidal categories}

Recall that a symmetric monoidal category is a category $\mathcal{M}$ equipped with a unital, associative and commutative tensor product

$$
\otimes: \mathcal{M} \times \mathcal{M} \longrightarrow \mathcal{M}
$$

In the sequel, 1 denotes the unit of this tensor product. The category $\mathcal{M}$ is $k$-linear, if $\mathcal{M}$ is an additive category and if the hom sets are $k$-vector spaces. In this appendix, a $k$-linear category is also assumed to be abelian. A $k$-linear monoidal category is a $k$-linear category equipped with a bilinear tensor product. The category $\mathcal{M}$ is graded, if each object has a canonical decomposition

$$
X=\bigoplus_{n \geq 0} X_{n}
$$

The tensor product is graded (in which case we call $\mathcal{M}$ a graded monoidal category), if the decomposition of $X \otimes Y$ is given by

$$
(X \otimes Y)_{n}=\bigoplus_{i+j=n} X_{i} \otimes Y_{j}
$$

Moreover, we assume that the unit for the tensor product is concentrated in degree 0 .

As mentionned in 1.1.3 and 2.2, the category of $\mathbf{S}$-modules and the category of 
right $\mathcal{P}$-modules are endowed with the structure of a graded $k$-linear symmetric monoidal category.

In this appendix, we show that the theory of Hopf algebras in the category of graded $\mathbf{Q}$-modules can be extended to any graded $\mathbf{Q}$-linear symmetric monoidal category.

\section{A.2. Coalgebras in a monoidal category}

A cocommutative (and coassociative) coalgebra in $\mathcal{M}$ is an object $C \in \mathcal{M}$ equipped with a coproduct $\Delta: C \longrightarrow C \otimes C$ and an augmentation $\epsilon: C \longrightarrow 1$. These morphisms are assumed to verify the classical relations. Namely, the coproduct $\Delta$ is coassociative and cocommutative and the augmentation $\epsilon$ is a counit for $\Delta$. The coalgebra $C$ is called connected if $C_{0}=1$. In this case, the degree 0 component of the coproduct

$$
C_{0} \stackrel{\Delta}{\longrightarrow}(C \otimes C)_{0}=C_{0} \otimes C_{0}
$$

is given by the canonical isomorphism $1=1 \otimes 1$.

\section{A.3. Construction of the cofree cocommutative coalgebra}

An object $V \in \mathcal{M}$ is connected, if $V_{0}=0$. In this case, we give an explicit construction of the cofree cocommutative coalgebra generated by $V$. More explicitly, we define a coalgebra $C(V)$ together with a canonical morphism $\pi: C(V) \longrightarrow V$ and we show that $C(V)$ verifies the following universal property. Given any cocommutative coalgebra $C$ equipped with a morphism $\pi^{\prime}: C \longrightarrow V$, there exists a unique morphism of coalgebras $\phi: C \longrightarrow C(V)$ such that $\pi \phi=\pi^{\prime}$.

Since $\mathcal{M}$ is symmetric monoidal, the tensor power $V^{\otimes n}$ is equipped with a canonical action of the symmetric group $S_{n}$. Let $C(V):=\oplus_{n \geq 0}\left(V^{\otimes n}\right)^{S_{n}}$. We equip $C(V)$ with the deconcatenation coproduct, whose components are given by the canonical embeddings

$$
\left(V^{\otimes n}\right)^{S_{n}} \hookrightarrow\left(V^{\otimes p}\right)^{S_{p}} \otimes\left(V^{\otimes q}\right)^{S_{q}} .
$$

Let $\pi: C(V) \longrightarrow V$ be the projection onto the linear component of $C(V)$.

A.4. Proposition. Let $V$ be a connected object in $\mathcal{M}$. The coalgebra defined above $C(V) \in \mathcal{M}$ is the cofree cocommutative coalgebra generated by $V$.

Proof. Fix a cocommutative coalgebra $C$ together with a morphism $\pi^{\prime}: C \longrightarrow V$.

1) Since $C$ is cocommutative, the iterated coproduct of $C$ induces a morphism $\Delta^{(r)}: C \longrightarrow\left(C^{\otimes r}\right)^{S_{r}}$. Let $\phi_{r}: C \longrightarrow\left(V^{\otimes r}\right)^{S_{r}}$ be the composite

$$
C \stackrel{\Delta^{(r)}}{\longrightarrow}\left(C^{\otimes r}\right)^{S_{r}} \stackrel{\pi^{\prime \otimes r}}{\longrightarrow}\left(V^{\otimes r}\right)^{S_{r}} .
$$


Since $V_{0}=0$, the map $\phi_{r}$ vanishes over the components $C_{i}$ of degree $i<r$. Therefore, we have a morphism $\phi: C \longrightarrow C(V)$ defined by $\phi=\sum \phi_{r}$. It is easy to see that $\phi$ is a morphism of coalgebras.

2) The composite

$$
C(V) \stackrel{\Delta^{(r)}}{\longrightarrow}\left(C(V)^{\otimes r}\right)^{S_{r}} \stackrel{\pi^{\otimes r}}{\longrightarrow}\left(V^{\otimes r}\right)^{S_{r}}
$$

is clearly equal to the projection $C(V) \longrightarrow\left(V^{\otimes r}\right)^{S_{r}}$. Therefore, if $\psi: C \longrightarrow$ $C(V)$ is a morphism of coalgebras, then the composite of $\psi$ with the projection $C(V) \longrightarrow\left(V^{\otimes r}\right)^{S_{r}}$ is equal to the composite $\pi^{\otimes r} \cdot \phi^{\otimes r} \cdot \Delta^{(r)}$. Hence, if $\pi \psi=\pi^{\prime}$, then we have necessarily $\psi=\phi$.

\section{A.5. Hopf algebras in a graded monoidal category}

Recall that an associative algebra in $\mathcal{M}$ is an object $A \in \mathcal{M}$ equipped with an associative product $m: A \otimes A \longrightarrow A$ and a unit $\eta: 1 \longrightarrow A$.

A (cocommutative) Hopf algebra in $\mathcal{M}$ is an object $H \in \mathcal{M}$ equipped with a (cocommutative) coalgebra structure and with a compatible algebra structure. More precisely, the algebra product $m: H \otimes H \longrightarrow H$ and the unit $\eta: 1 \longrightarrow H$ are supposed to be morphisms of coalgebras. (As for classical coalgebras, the tensor product in $\mathcal{M}$ of coalgebras is equipped with a canonical coalgebra structure.) Moreover, we assume that $H$ is connected. This condition ensures the existence of an antipode $s: H \longrightarrow H$.

As for classical coalgebras, the product in the category of cocommutative coalgebras in $\mathcal{M}$ is given by the tensor product of $\mathcal{M}$. As a consequence, a cocommutative Hopf algebra in $\mathcal{M}$ is equivalent to a group in the category of coalgebras in $\mathcal{M}$.

\section{A.6. The enveloping algebra of a Lie algebra in a graded monoidal cat- egory}

A Lie algebra in $\mathcal{M}$ is an object $L \in \mathcal{M}$ equipped with a product $\gamma: L \otimes L \longrightarrow L$ (the Lie bracket) which is antisymmetric and verifies the Jacobi identity

$$
\gamma \cdot 1 \otimes \gamma \cdot\left(1+c_{*}+c_{*}^{2}\right)=0
$$

where $c_{*}$ denotes the action of the cycle $c=\left(\begin{array}{lll}1 & 2 & 3\end{array}\right)$ on $L^{\otimes 3}$.

If $A$ is an associative algebra in $\mathcal{M}$, then by antisymmetrization of the algebra product $m: A \otimes A \longrightarrow A$, we equip $A$ with a canonical structure of a Lie algebra in $\mathcal{M}$. Explicitly, if $\tau_{*}$ denotes the action of the transposition $\tau=\left(\begin{array}{ll}1 & 2\end{array}\right)$ on $A^{\otimes 2}$, then the Lie bracket of $A$ is given by the formula $\gamma=m-m \tau_{*}$. As in the classical theory, this Lie-ification functor admits a left adjoint known as the enveloping algebra functor. We denote by $U(L)$ the enveloping algebra of $L$. It is easy to generalize the construction of the enveloping algebra of a classical Lie algebra to our context. The tensor algebra in $\mathcal{M}$ is the object $T(L)=\oplus_{n} L^{\otimes n}$ equipped with 
the concatenation product. The algebra $U(L)$ is the quotient of $T(L)$ by the ideal generated by the relations

$$
m-m \tau_{*}=\gamma,
$$

where $m: L \otimes L \longrightarrow T(L)$ is induced by the concatenation product and $\gamma:$ $L \otimes L \longrightarrow T(L)$ is induced by the Lie bracket of $L$.

The tensor algebra $T(L)$ is equipped with a Hopf algebra structure. The component $L^{\otimes n} \longrightarrow L^{\otimes p} \otimes L^{\otimes q}$ of the coproduct of $T(L)$ is the sum of all $(p, q)$-shuffles. The shuffle coproduct induces a coproduct on $U(L)$ and hence makes $U(L)$ into a Hopf algebra in $\mathcal{M}$.

Let $V \in \mathcal{M}$. The shuffle coproduct equips $S(V)=\oplus_{n}\left(V^{\otimes n}\right)_{S_{n}}$ with a coalgebra structure. Let $i: L \longrightarrow U(L)$ denotes the canonical morphism. By symmetrization, the sum of the composites

$$
L^{\otimes n} \stackrel{i^{\otimes n}}{\longrightarrow} U(L) \stackrel{\otimes n}{\text { Product }} \longrightarrow U(L)
$$

induces

$$
S(L) \longrightarrow U(L)
$$

The classical Poincaré-Birkhoff-Witt and Milnor-Moore theorems can be extended without changes to any graded $\mathbf{Q}$-linear monoidal category:

A.7. Theorem (Poincaré-Birkhoff-Witt). Assume that $\mathcal{M}$ is a graded $\mathbf{Q}$-linear monoidal category. Let $L$ be a Lie algebra in $\mathcal{M}$. The map $S(L) \longrightarrow U(L)$ introduced above is an isomorphism of coalgebras.

Let $H$ be any Hopf algebra in $\mathcal{M}$. The primitive part of $H$ is defined as the equalizer

$$
\operatorname{Prim} H \longrightarrow H \underset{1 \otimes \eta+\eta \otimes 1}{\stackrel{\Delta}{\longrightarrow}} H \otimes H .
$$

Let $\gamma$ denote the antisymmetrization of the product of $H$. As in the classical theory, one verifies easily that $H$ is stable under $\gamma$. Therefore, Prim $H$ is endowed with a Lie algebra structure.

A.8. Theorem (Milnor-Moore). Assume that $\mathcal{M}$ is a graded $\mathbf{Q}$-linear monoidal category.

(1) Let $H$ be a connected cocommutative Hopf algebra in the monoidal category M. The embedding Prim $H \longrightarrow H$ induces a morphism of associative algebras 
in $\mathcal{M}$

$$
e: U(\operatorname{Prim} H) \longrightarrow H
$$

which is an isomorphism of Hopf algebras.

(2) Let $L$ be a Lie algebra in the monoidal category $\mathcal{M}$. The canonical morphism $L \longrightarrow U(L)$ induces a morphism of Lie algebras in $\mathcal{M}$

$$
i: L \longrightarrow \operatorname{Prim} U(L)
$$

which is an isomorphism.

We refer to the article [20]. The demonstration of the Poincaré-Birkhoff-Witt theorem and of the Milnor-Moore theorem given in this article works in any graded Q-linear monoidal category. If $\mathcal{M}$ is abelian semi-simple, then it is also possible to translate the classical demonstration of the Poincaré-Birkhoff-Witt theorem and of the Milnor-Moore theorem (see for instance [21, Appendix B]). Hence, the classical demonstration works in the context of $\mathbf{S}$-modules. It is also immediate to deduce theorems A.7 and A.8 for $\mathcal{P}$-linear Hopf algebras from the case of $\mathbf{S}$-modules. We refer to [24] for a proof of theorems A.7 and A.8 for S-modules valid over a field of positive characteristic.

There is a transfer morphism $\left(V^{\otimes n}\right)_{S_{n}} \longrightarrow\left(V^{\otimes n}\right)^{S_{n}}$ induced by the action of the trace $\sum_{S_{n}} \sigma \in k\left[S_{n}\right]$ on the tensor power $V^{\otimes n}$. Clearly, the transfer induces a morphism of coalgebras from $S(V)$ to $C(V)$. If $\mathcal{M}$ is $\mathbf{Q}$-linear, then the transfer is an isomorphism, and hence, $S(V)$ is a realization of the cofree coalgebra functor. Therefore, from theorem A.8 and A.7, we deduce:

A.9. Theorem. Assume that $\mathcal{M}$ is a graded $\mathbf{Q}$-linear monoidal category. If $H$ is a connected cocommutative Hopf algebra in the monoidal category $\mathcal{M}$, then $H$ is necessarily a cofree coalgebra. More precisely, the embedding Prim $H \hookrightarrow H$ extends canonically to an isomorphism of coalgebras $C(\operatorname{Prim} H) \stackrel{\longrightarrow}{\longrightarrow} H$.

\section{Acknowledgement}

I thank the referee for his careful reading of the manuscript and for his accurate remarks, which make the exposition less confused.

\section{References}

[1] I. Berstein, On cogroups in the category of graded algebras, Trans. Amer. Math. Soc. 115 (1965), 257-269.

[2] P. Cartier, Groupes algébriques et groupes formels. In: Colloque sur la théorie des groupes 
algébriques, Bruxelles, CBRM, (1962), 87-112.

[3] C. Cuvier, Homologie des algèbres de Leibniz, Ann. Sci. Ecole Norm. Sup. 27 (1994), 1-45.

[4] B. Fresse, Lie theory of formal groups over an operad, preprint I.R.M.A., Strasbourg, 1996.

[5] E. Getzler, J.D.S. Jones, Operads, homotopy algebra and iterated integrals for double loop spaces, preprint (1994).

[6] V. Ginzburg, M.M. Kapranov, Koszul duality for operads, Duke Math. J. 76 (1995), 203-272.

[7] C.U. Jensen, On the vanishing of $\lim ^{(i)}$, J. Algebra 15 (1970), 151-166.

[8] A. Joyal, Foncteurs analytiques et espèces de structures, Lecture Notes in Math. 1234, Springer-Verlag, 1986, 126-159.

[9] M. Kontsevich, Formal (non)-commutative symplectic geometry. In: The Gelfand mathematical seminars, 1990-1992, Birkhäuser 1993, 173-187.

[10] M. Lazard, Lois de groupes et analyseurs, Ann. Sci. Ecole Norm. Sup. 62 (1955), 299-400.

[11] J.-L. Loday, Cyclic Homology, Springer-Verlag, 1992.

[12] J.-L. Loday, La renaissance des opérades, Séminaire Bourbaki, Exp. 792, 1994-1995.

[13] J.-L. Loday, D. Quillen, Cyclic homology and the Lie algebra homology of matrices, Comment. Math. Helvetici. 59 (1984), 565-591.

[14] S. Mac Lane, Categories for the Working Mathematician, Springer Verlag, 1971.

[15] M. Markl, Models for operads, Comm. Alg. 24 (1996), 1471-1500.

[16] M. Markl, Simplex, associahedron, and cyclohedron, preprint (1996).

[17] M. Markl, A compactification of the real configuration space as an operadic completion, preprint (1996). (http://xxx.lanl.gov/abs/hep-th/9608067)

[18] J.P. May, The geometry of iterated loop spaces, Lecture Notes in Math. 271, SpringerVerlag, 1972.

[19] J.-M. Oudom, Coproduct and cogroups in the category of graded dual Leibniz algebras, preprint (1995).

[20] F. Patras, L'algèbre des descentes d'une bigèbre graduée, J. Algebra 170 (1994), 547-566.

[21] D. Quillen, Rational homotopy theory, Ann. of Math. 90 (1969), 205-295.

[22] C. Reutenauer, Free Lie Algebras, London Math. Soc. Mon. 7, Clarendon Press, 1993.

[23] V.A. Smirnov, Homotopy theory of coalgebras, Izv. Akad. Nauk SSSR Ser. Mat. 49 (1985), 1302-1321 (in Russian). Math. USSR Izv. 27 (1986), 575-592 (English translation).

[24] C. Stover, The equivalence of certain categories of twisted Lie and Hopf algebras over a commutative ring, J. Pure Appl. Algebra 86 (1993), 289-326.

[25] M.E. Sweedler, Hopf algebras, Benjamin, 1969.

[26] B.L. Tsygan, The homology of matrix Lie algebras over rings and the Hochschild homology, Uspekhi Mat. Nauk. 38 (1983), 217-218 (in Russian). Russ. Math. Survey 38 (1983), 198-199 (English translation).

Benoit Fresse

Laboratoire J.A. Dieudonné

Université de Nice

Parc Valrose

F-06108 Nice Cedex 02

France

e-mail: fresse@math.unice.fr

(Received: August 26, 1996 and final version, February 4, 1998) 